\title{
DIS-CARD: a new method of multiple criteria sorting to classes with desired cardinality
}

\author{
Miłosz Kadziński · Roman Słowiński
}

Received: 17 August 2011 / Accepted: 13 June 2012 / Published online: 4 July 2012

(C) The Author(s) 2012. This article is published with open access at Springerlink.com

\begin{abstract}
In this paper, we present a new preference disaggregation method for multiple criteria sorting problems, called DIS-CARD. Real-life experience indicates the need of considering decision making situations in which a decision maker (DM) specifies a desired number of alternatives to be assigned to single classes or to unions of some classes. These situations require special methods for multiple criteria sorting subject to desired cardinalities of classes. DIS-CARD deals with such a problem, using the ordinal regression approach to construct a model of DM's preferences from preference information provided in terms of exemplary assignments of some reference alternatives, together with the above desired cardinalities. We develop a mathematical model for incorporating such preference information via mixed integer linear programming (MILP). Then, we adapt the MILP model to two types of preference models: an additive value function and an outranking relation. Illustrative example is solved to illustrate the methodology.
\end{abstract}

Keywords Multiple criteria sorting · Preference disaggregation · Desired class cardinality · Additive value function · Outranking relation

\section{Introduction}

Multiple criteria sorting problems involve an assignment of a set of alternatives evaluated on a family of criteria into homogeneous classes which are given in a preference order. The classes have a semantic definition and are pre-defined, which means that they do not result from the analysis. Such a discrimination among two or more sets of alternatives is a problem of a major practical interest in such fields as finance (e.g., long term obligation rating for

M. Kadziński $(\varangle) \cdot$ R. Słowiński

Institute of Computing Science, Poznań University of Technology, 60-965 Poznan, Poland

e-mail: milosz.kadzinski@cs.put.poznan.pl

R. Słowiński

Systems Research Institute, Polish Academy of Sciences, 01-447 Warsaw, Poland

e-mail: roman.slowinski@cs.put.poznan.pl 
government entities, business failure prediction, or credit risk assessment), medicine (e.g., groups of patients at different risk levels), human resource management (e.g., portfolio selection), or environmental protection (e.g., analysis of environmental impacts of different energy policies).

For decades, sorting problems were approached only with statistical discrimination methods. However, since 1980s one observes landmark research in multiple criteria decision aiding (MCDA) sorting methods [26]. This research was focused on the development of preference modeling procedures that enable incorporation of the DM's preference information into the sorting model. Precisely, preference information elicited from the DM, and considered in various approaches, is either direct or indirect. Direct preference information specifies parameters of the employed preference model and limiting or central profiles (see, e.g., $[1,20,24])$ of the classes defined in the criteria space, while indirect preference information is a set of exemplary assignments of some reference alternatives to particular classes or to unions of some classes (see, e.g., $[15,21,25]$ ). The former may refer, e.g., to substitution rates among criteria in an additive linear value function model considered within multi-attribute value theory (MAVT) [14], or to weights of the criteria and comparison thresholds used in the outranking approach [22]. The latter is necessary for finding the parameters of preference models used for sorting within either MAVT or the outranking approach, via a disaggregation procedure called ordinal regression [23].

Although in many real-world sorting problems it is necessary to take into account requirements with respect to the desired cardinality of classes, these problems did not receive due attention in MCDA research. In fact, people often express this type of requirements, using statements like the following ones: "we wish to accept at least 5 and not more than 10 candidates", "we need to reject at least 30 applications and not more than 20 others may deserve further consideration", "at most $10 \%$ of the employees can be provided with the highest incentive package", or "the cardinalities of all classes needs to be balanced". Note that these are most often imprecise statements which refer to the lower and upper limits on the number of alternatives to be assigned to a particular class or to unions of some classes. These bounds are specified either as explicit numbers or as a percent of the whole set of alternatives.

In this paper, we present a method, called DIS-CARD, which handles the above requirements about desired cardinalities of classes. In DIS-CARD, this type of information is combined with "traditional" preference information provided in a direct way, but with some tolerated imprecision, or in an indirect way, i.e. with assignment examples. The latter type of preference informations makes that DIS-CARD is based on the ordinal regression approach. Using the ordinal regression approach, one gets, in general, a rich set of compatible instances of the assumed preference model, and, consequently, one can use such a compatible instance which best satisfies the required cardinalities of classes. Compatible instance means here a set of parameter values which ensures that the assumed preference model represents (is concordant with) the preference information provided by the DM. Note that the requirement of imprecise or indirect preference information is an advantage of the proposed method, because it is easier to provide for the DM than precise and direct preference information.

In the literature, there is known only one work [18] close to the approach proposed in this paper. This work, however, is concerned with more formal discussion on the so-called "Constrained Sorting Problem" (CSP). In particular, in [18] the authors introduce the notion of "the category size" defined as "the proportion by which an evaluation vector corresponding to a realistic alternative is assigned to the category". They consider the cases when a complete description of the set of alternatives $A$ is available (static CSP) or when $A$ is imprecisely known beforehand (anticipatory CSP). They also discuss ways of expressing constraints on category sizes for both static and anticipatory CSPs. Finally, they propose a procedure for 
inferring the values for preference model parameters that accounts for the preference about the size of categories in the context of the UTADIS method [4].

DIS-CARD is focused instead on adapting some well-known MCDA sorting methods to deal specifically with desired cardinalities of classes. In particular, assuming an additive value function preference model, apart from considering a threshold-based sorting procedure as in UTADIS [4], we propose also an example-based sorting procedure as in $[8,17]$. Moreover, we adapt our proposal to some popular outranking methods, which are based on assignment examples [15], limiting class profiles [20,24], or central and typical class profiles [1,2]. In this way, DIS-CARD is concerned with the preference modeling point of view rather than with some theoretical considerations and ordinal regression formulation concerning a single method (UTADIS). We show that the preference information concerning the desired cardinalities of classes can be translated into mathematical constraints which are irrespective of the underlying method. Moreover, we suitably adapt (in case of value-based methods) or newly introduce (in case of outranking-based methods) disaggregation procedures which make it possible to account for this type of preference information. Finally, we present some procedures for selection of a single preference model instance compatible with the requirements concerning the desired class cardinalities. We also discuss the way of computing all possible assignments for each alternative. Although our proposal is focused on the case where both assignment examples and final recommendation for each alternative are single class assignments, when discussing an example-based value-driven sorting procedure, we show how DIS-CARD can be extended to the case of assignment examples and recommendation concerning an interval of contiguous classes.

The organization of the paper is the following. In the next section, we introduce basic concepts and notation that will be used in the paper. We also outline existing multiple criteria sorting methods and define the models that we refer to in our approach. In Sect. 3, we present the mathematical model which handles the preference information referring to the desired cardinalities of classes. In Sect. 4, we discuss how to choose a single instance of the preference model compatible with the new type of preference information considered in this paper. Section 5 provides an illustrative example showing a practical application of DIS-CARD. The last section concludes the paper.

\section{Concepts and notation}

We shall use the following notation:

- $A=\left\{a_{1}, a_{2}, \ldots, a_{i}, \ldots, a_{n}\right\}$ - a finite set of $n$ alternatives;

- $A^{R}=\left\{a^{*}, b^{*}, \ldots\right\}-$ a finite set of reference alternatives, on which the DM accepts to express preferences; usually, $A^{R} \subseteq A$;

- $G=\left\{g_{1}, g_{2}, \ldots, g_{j}, \ldots, g_{m}\right\}$-a finite set of $m$ evaluation criteria, $g_{j}: A \rightarrow \mathbb{R}$ for all $j \in J=\{1,2, \ldots, m\}$

- $X_{j}=\left\{g_{j}\left(a_{i}\right), a_{i} \in A\right\}$ - the set of all different evaluations on $g_{j}, j \in J$; we assume, without loss of generality, that the greater $g_{j}\left(a_{i}\right)$, the better alternative $a_{i}$ on criterion $g_{j}$, for all $j \in J$;

- $x_{j}^{1}, x_{j}^{2}, \ldots, x_{j}^{n_{j}(A)}$ - the ordered values of $X_{j}, x_{j}^{k}<x_{j}^{k+1}, k=1,2, \ldots, n_{j}(A)-1$, where $n_{j}(A)=\left|X_{j}\right|$ and $n_{j}(A) \leq n$; consequently, $X=\prod_{j=1}^{m} X_{j}$ is the evaluation space;

- $C_{1}, C_{2}, \ldots, C_{p}$ - predefined preference ordered classes, each having a specific semantic definition; $C_{h+1}$ is preferred to $C_{h}, h=1, \ldots, p-1$.

Table 1 summarizes the notation used throughout the paper. 
2.1 Value-driven sorting procedures

When approaching sorting problems with the use of MAVT [14], we shall employ a preference model in the form of an additive value function:

$$
U(a)=\sum_{j=1}^{m} u_{j}\left(g_{j}(a)\right)=\sum_{j=1}^{m} u_{j}(a)
$$

with general marginal value functions $u_{j}$, taking values $u_{j}\left(x_{j}^{k}\right), k=1, \ldots, n_{j}(A), j \in J$, in characteristic points. Marginal value functions are monotone non-decreasing and normalized, so that comprehensive value $U$ is bounded to the interval $[0,1]$, i.e.:

$$
\left.\begin{array}{l}
u_{j}\left(x_{j}^{k}\right)-u_{j}\left(x_{j}^{(k-1)}\right) \geq 0, j \in J, k=2, \cdots, n_{j}(A), \\
u_{j}\left(x_{j}^{1}\right)=0, j \in J, \\
\sum_{j=1}^{m} u_{j}\left(x_{j}^{n_{j}(A)}\right)=1 .
\end{array}\right\} E_{\text {base }}^{\mathrm{UD}}
$$

The first method based on the ordinal regression approach, that was designed for multiple criteria sorting problems, and that employs an additive value function, was UTADIS (see, e.g., $[4,25])$. The preference information supplied by the DM is a set of assignment examples on a subset of reference alternatives $A^{R}$. Each assignment example specifies a desired assignment of a corresponding reference alternative $a^{*} \in A^{R}$ to a single class:

$$
a^{*} \rightarrow C_{h^{\mathrm{DM}}\left(a^{*}\right)} \text {. }
$$

The original UTADIS used a threshold-based sorting procedure, where each class $C_{h}, h=$ $1, \ldots, p$, is delimited by the lower $b_{h-1}$ and upper $b_{h}$ thresholds defined on the comprehensive value $U$. These thresholds are equal to the infimum and supremum values for an alternative to be assigned to class $C_{h}$, respectively. A pair $(U, \mathbf{b})$, where $U$ is a value function and $\mathbf{b}=\left[b_{1}, \ldots, b_{p-1}\right]$ is a vector of thresholds $b_{h}, h=1, \ldots, p-1$, such that $0<b_{1}<b_{2}<\cdots<b_{p-1}<1$, is consistent with the preference information provided by the DM, if for all $a^{*} \in A^{R}$, such that $a^{*} \rightarrow C_{h^{\mathrm{DM}}\left(a^{*}\right)}$ :

$$
b_{h^{\mathrm{DM}}\left(a^{*}\right)}>U\left(a^{*}\right) \geq b_{h^{\mathrm{DM}}\left(a^{*}\right)-1} .
$$

Consequently, the set of constraints which represents preference information given in the form of assignment examples is the following:

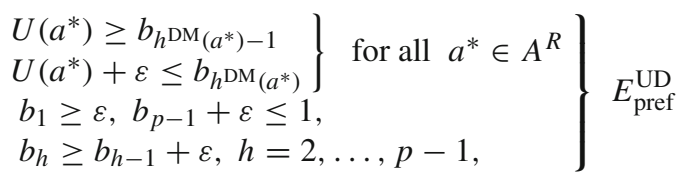

where $\varepsilon$ is a small positive value (we will use this interpretation of $\varepsilon$ in all below formulations). In order to achieve higher discriminating and predicting ability, Doumpos and Zopounidis [25] proposed to calculate an additive value function and the values of thresholds delimiting the classes, by maximizing the minimum distance of the comprehensive values of reference alternatives from the class thresholds.

The UTADIS method was generalized in [10] and [15] by allowing imprecise assignments, and by taking into account the whole set of compatible additive value functions as DM's preference model; in consequence, the DM gets information about the range of possible classes to which each alternative can be assigned. Köksalan and Özpeynirci [15] consider marginal value functions which are piecewise linear, as in the original UTADIS, whereas Greco et al. 
Table 1 Notation

\begin{tabular}{|c|c|}
\hline Notation & Meaning \\
\hline$E_{Y}^{X}$ & $\begin{array}{l}\text { Set of constraints which models } Y \text { for method } X \\
\mathrm{X} \in\{\mathrm{UD}, \mathrm{UE}, \mathrm{EL}, \mathrm{EBP}, \mathrm{EBO}, \mathrm{EC}, \mathrm{ECD}, \mathrm{ECA}, \mathrm{EnCD}, \mathrm{EnCA}, \mathrm{PR}\} \\
\mathrm{Y} \in\{\text { base, pref, card, post-opt }\}\end{array}$ \\
\hline UD & UTADIS \\
\hline UE & Assignment-based value-driven sorting procedure \\
\hline EL & ELECTRE \\
\hline EBP & Pessimistic procedure of ELECTRE TRI \\
\hline $\mathrm{EBO}$ & Optimistic procedure of ELECTRE TRI \\
\hline $\mathrm{EC}$ & ELECTRE-like assignment-based procedure \\
\hline ECD & Descending rule of ELECTRE TRI-C \\
\hline ECA & Ascending rule of ELECTRE TRI-C \\
\hline $\mathrm{EnCD}$ & Descending rule of ELECTRE TRI-nC \\
\hline EnCA & Ascending rule of ELECTRE TRI-nC \\
\hline PR & FlowSort \\
\hline Base & $\begin{array}{l}\text { Monotonicity of marginal functions and normalization } \\
\text { of the comprehensive scores (values, indices, or flows) }\end{array}$ \\
\hline Pref & Preferences in form of assignment examples \\
\hline Card & Desired cardinalities of classes \\
\hline Post-opt & Optimization criteria for post-optimality analysis \\
\hline$U(a)$ & Comprehensive value of alternative $a \in A$ \\
\hline$u_{j}(a)$ & Marginal value of alternative $a \in A$ on criterion $g_{j}$ \\
\hline$C_{h^{\mathrm{DM}}}\left(a^{*}\right)$ & Desired precise assignment for reference alternative $a^{*} \in A^{R}$ \\
\hline$\left[C_{L^{U}(a)}, C_{R^{U}(a)}\right]$ & Assignment of $a$ with a value function $U$ \\
\hline$C(a, b)$ & Concordance index for ordered pair $(a, b) \in A \times A$ \\
\hline$\psi_{j}(a, b)$ & Marginal concordance index for ordered pair $(a, b) \in A \times A$ \\
\hline$d_{j}(a, b)$ & Marginal discordance index for ordered pair $(a, b) \in A \times A$ \\
\hline$q_{j}$ & Indifference threshold for criterion $g_{j}$ \\
\hline$p_{j}$ & Preference threshold for criterion $g_{j}$ \\
\hline$v_{j}$ & Veto threshold for criterion $g_{j}$ \\
\hline$k_{j}$ & Weight of criterion $g_{j}$ \\
\hline$\left[q_{j, \text { inf }}, q_{j}^{\text {sup }}\right]$ & Value interval specified by the DM for indifference threshold $q_{j}$ \\
\hline$\left[p_{j, i n f}, p_{j}^{s u p}\right]$ & Value interval specified by the DM for preference threshold $p_{j}$ \\
\hline$\lambda$ & Concordance (cutting) level \\
\hline$S$ & Outranking relation \\
\hline$S^{c}$ & Non-outranking relation \\
\hline$v_{a, C_{h}}$ & Binary variable corresponding to assignment of $a$ to $C_{h}$ \\
\hline$C_{h}^{\min }$ & Desired minimum cardinality of class $C_{h}$ \\
\hline$C_{h}^{\max }$ & Desired maximum cardinality of class $C_{h}$ \\
\hline$C_{h}^{\min -\text { perc }}($ in $\%)$ & Desired minimum cardinality (in $\%$ of the cardinality of $A$ ) of class $C_{h}$ \\
\hline$C_{h}^{\max -p e r c}($ in \%) & Desired maximum cardinality (in $\%$ of the cardinality of $A$ ) of class $C_{h}$ \\
\hline
\end{tabular}


Table 1 continued

\begin{tabular}{ll}
\hline Notation & Meaning \\
\hline$C_{h, l}^{\min }$ & Desired minimum cardinality of union of classes $C_{h}, C_{h+1}, \ldots, C_{l}$ \\
$C_{h, l}^{\max }$ & Desired maximum cardinality of union of classes $C_{h}, C_{h+1}, \ldots, C_{l}$ \\
$a_{j}^{*}\left(a_{j, *}\right)$ & Alternative $a \in A$ with the best (worst) evaluation on $g_{j}$ \\
\hline
\end{tabular}

employ in the UTADIS GMS method [10] general monotone value functions and, additionally, propose to compute necessary assignments which specify the most certain recommendations worked out on the basis of all compatible value functions considered simultaneously. Both methods are interactive in the sense that during the solution process the DM is occasionally required to place some additional reference alternatives into classes, that affects classification of the remaining alternatives as well.

When it comes to an example-based value driven sorting procedure, classes are implicitly delimited by some assignment examples rather than threshold on a utility scale (see, e.g., $[11,17])$. Precisely, such a procedure is driven by a value function $U$ and its associated assignment examples. The procedure assigns an alternative $a \in A$ to an interval of classes $\left[C_{L^{U}(a)}, C_{R^{U}(a)}\right]$, in the following way (note that we assume precise assignment examples):

$$
\begin{aligned}
& L^{U}(a)=\operatorname{Max}\left\{h^{\mathrm{DM}}\left(a^{*}\right): U\left(a^{*}\right) \leq U(a), a^{*} \in A^{R}\right\}, \\
& R^{U}(a)=\operatorname{Min}\left\{h^{\mathrm{DM}}\left(a^{*}\right): U\left(a^{*}\right) \geq U(a), a^{*} \in A^{R}\right\} .
\end{aligned}
$$

In particular, if there is at least one assignment example for each class, then each non-reference alternative can be assigned either to one or two consecutive classes. When using such a procedure, the consistency of a value function $U$ with a set of assignment examples could be modeled in the following way:

$$
\left.\begin{array}{l}
U\left(a^{*}\right) \geq U\left(b^{*}\right)+\varepsilon, \\
\quad \text { for all } a^{*}, b^{*} \in A^{R}, \text { such that } h^{\mathrm{DM}}\left(a^{*}\right)>h^{\mathrm{DM}}\left(b^{*}\right) .
\end{array}\right\} E_{\mathrm{pref}}^{\mathrm{UE}}
$$

\subsection{ELECTRE-like methods}

When approaching sorting problems with the use of outranking methods [22], we shall employ a preference model in the form of an outranking relation which becomes true when a concordance index is greater than some cutting level. We will also not take into account the veto phenomenon. In order to construct an outranking model, we assume that the intra-criterion preference information concerning indifference and preference thresholds $p_{j} \geq q_{j} \geq 0, j \in J$, respectively, is given. Note that when comparing two alternatives $a, b \in A$ on a given criterion, the zone between indifference and preference thresholds corresponds to hesitation between opting for indifference and preference. We admit that the DM provides a real interval for each threshold, rather than a precise value. We use the following notation to represent these intervals:

- $\left[q_{j, \text { inf }}, q_{j}^{\text {sup }}\right]$, where $q_{j, \text { inf }}$ and $q_{j}^{\text {sup }}$ are, respectively, the least and the greatest value of indifference threshold allowed by the DM,

- $\left[p_{j, i n f}, p_{j}^{\text {sup }}\right]$, where $p_{j, \text { inf }}$ and $p_{j}^{\text {sup }}$ are, respectively, the least and the greatest value of preference threshold allowed by the DM. 
The concordance index represents the strength of the coalition of criteria being in favor of the statement " $a$ outranks $b$ " $(a S b)$. It is defined in the following way:

$$
C(a, b)=\sum_{j=1}^{m} \psi_{j}(a, b),
$$

where the marginal concordance indices $\psi_{j}(a, b), j \in J$, are defined as in [7] and [13], i.e., they are general non-decreasing real-valued functions with clearly distinguished zones of strict and weak preference as well as indifference, and not only piecewise linear or of other arbitrarily chosen shape. We are bounding, however, the area of variation of a marginal concordance index in the zone of weak preference using some extreme linear shapes of this index, which are consistent with the intra-criterion preference information provided by the DM. Moreover, we assume that no criterion is more important than all the other criteria jointly. Consequently, the compatible marginal concordance indices are defined by the following set of constraints:

$$
\begin{aligned}
& \sum_{j=1}^{m} \psi_{j}\left(a_{j}^{*}, a_{j, *}\right)=1, \text { for all } j \in J: \\
& \left(g_{j}\left(a_{j}^{*}\right)=x_{j}^{n_{j}(A)}\right) \text { and }\left(g_{j}\left(a_{j, *}\right)=x_{j}^{1}\right) \text { with } a_{j}^{*}, a_{j, *} \in A, \\
& \psi_{j}\left(a_{j}^{*}, a_{j, *}\right) \leq 0.5, j \in J, \\
& \text { for all }(a, b, c, d) \in A \times A \times A \times A, j \in J: \\
& \quad \psi_{j}(a, b) \geq \psi_{j}(c, d) \text { if } g_{j}(a)-g_{j}(b)>g_{j}(c)-g_{j}(d), \\
& \psi_{j}(a, b)=\psi_{j}(c, d) \text { if } g_{j}(a)-g_{j}(b)=g_{j}(c)-g_{j}(d), \\
& \text { for all }(a, b) \in A \times A, j \in J: \\
& \quad \psi_{j}(a, b)=0 \text { if } g_{j}(a)-g_{j}(b) \leq-p_{j}^{\text {sup }}, \\
& \quad \psi_{j}(a, b) \geq \varepsilon \text { if } g_{j}(a)-g_{j}(b)>-p_{j, i n f}, \\
& \psi_{j}(a, b)=\psi_{j}\left(a_{j}^{*}, a_{j, *}\right) \text { if } g_{j}(a)-g_{j}(b) \geq-q_{j, i n f}, \\
& \quad \psi_{j}(a, b)+\varepsilon \leq \psi_{j}\left(a_{j}^{*}, a_{j, *}\right) \text { if } g_{j}(a)-g_{j}(b)<-q_{j}^{\text {sup }}, \\
& \quad \psi_{j}(a, b) \geq \psi_{j}\left(a_{j}^{*}, a_{j, *}\right) \cdot\left[p_{j, i n f}-\left(g_{j}(b)-g_{j}(a)\right)\right] /\left(p_{j, i n f}-q_{j, i n f}\right) \\
& \quad \text { if }-q_{j, \text { inf }}>g_{j}(a)-g_{j}(b)>-p_{j, \text { inf }}, \\
& \quad \psi_{j}(a, b) \leq \psi_{j}\left(a_{j}^{*}, a_{j, *}\right) \cdot\left[p_{j}^{\text {sup }}-\left(g_{j}(b)-g_{j}(a)\right)\right] /\left(p_{j}^{\text {sup }}-q_{j}^{\text {sup }}\right) \\
& \quad \text { if }-q_{j}^{\text {sup }}>g_{j}(a)-g_{j}(b)>-p_{j}^{\text {sup }} .
\end{aligned}
$$

If the compatible concordance indices would be used in a method that takes into account class profiles $B=\left\{b_{0}, b_{1}, \ldots, b_{p}\right\}$, then in the above formulation one should consider set $A \cup B$ instead of $A$ only. For a more detailed definition and explanation of each constraint, see [7]. Note that the lower and upper bounds for indifference and preference thresholds can be defined by means of non-decreasing functions taking $g_{j}(a)$ as an argument; then, they are denoted by $\left[q_{j, \text { inf }}(a), q_{j}^{\text {sup }}(a)\right]$ and $\left[p_{j, \text { inf }}(a), p_{j}^{\text {sup }}(a)\right]$. In particular, these can be affine functions which are used in most ELECTRE methods. Moreover, in case the DM is able to provide preference information about the weights of the criteria $k_{j}, j \in J$, we may represent the DM's preference statements in the following way:

- interval weights of the criteria $k_{j}$, i.e. $k_{j} \in\left[k_{j, *}, k_{j}^{*}\right] \Rightarrow \psi_{j}\left(a_{j}^{*}, a_{j, *}\right) \geq k_{j, *}$ and $\psi_{j}\left(a_{j}^{*}, a_{j, *}\right) \leq k_{j}^{*}$,

- pairwise comparisons of the weights of the criteria, e.g., $k_{i} \geq k_{j} \Rightarrow \psi_{i}\left(a_{i}^{*}, a_{i, *}\right) \geq$ $\psi_{j}\left(a_{j}^{*}, a_{j, *}\right)$.

We conclude that $a$ outranks $b$, if $C(a, b) \geq \lambda$, where $\lambda \in[0.5,1]$ is a concordance cutting level, and that $a$ does not outrank $b\left(a S^{c} b\right)$, otherwise. Knowing whether $S$ is true or not for 
an ordered pair of alternatives $(a, b) \in A \times A$, one is able to represent situations of weak $(Q)$ or strict $(P)$ preference, indifference $(\sim)$, and incomparability (?) among $a$ and $b$ :

$$
\begin{aligned}
& a S b \text { and } b S^{c} a \Leftrightarrow a Q b \text { or } a P b \Leftrightarrow a \succ b, \\
& \quad \text { where } \succ=\{Q \cup P\}, \\
& a S b \text { and } b S a \Leftrightarrow a \sim b, \\
& a S^{c} b \text { and } b S^{c} a \Leftrightarrow a ? b .
\end{aligned}
$$

The first outranking-based method for multiple criteria sorting problems was ELECTRE TRI [24]. It compares alternatives to some evaluation profiles which are considered as representative fictitious alternatives delimiting the classes from the top and from the bottom. The profiles are denoted by $b_{0}, b_{1}, \ldots, b_{p}$, with $b_{h}, h=1, \ldots, p-1$, being the upper profile of class $C_{h}$ and the lower profile of class $C_{h+1}$. The method verifies the truth of an outranking relation $S$ between pairs composed of alternatives and class profiles, and exploits this relation to assign each alternative to a specific class, using two assignment procedures. A pessimistic procedure compares $a$ successively to $b_{h}$, for $h=p-1, \ldots, 0$, and assigns it to class $C_{h+1}$, finding the first profile $b_{h}$, such that $a S b_{h}$. Using the terms of the model employed in this paper, $a$ is assigned to $C_{h}$, according to the pessimistic procedure, if the following set of constraints is satisfied:

$$
\left.\begin{array}{l}
C\left(a, b_{t}\right) \geq \lambda, \text { for } t=0, \ldots, h-1, \\
C\left(a, b_{t}\right)+\varepsilon \leq \lambda, \text { for } t=h, \ldots, p-1 .
\end{array}\right\}
$$

However, since ELECTRE TRI requires that $b_{t}$ dominates $b_{t-1}, t=1, \ldots, p$, the above set of conditions could be reduced to the following set of constraints:

$$
\left.\begin{array}{l}
C\left(a, b_{h-1}\right) \geq \lambda, \\
C\left(a, b_{h}\right)+\varepsilon \leq \lambda, \text { if } h<p .
\end{array}\right\}
$$

As far as an optimistic procedure is concerned, it compares $a$ successively to $b_{h}, h=$ $1, \ldots, p$, and assigns it to class $C_{h}$, such that $b_{h}$ is the first profile for which $b_{h} \succ a$. As a result, $a$ is assigned to $C_{h}$, if the following set of constraints is satisfied:

$$
\begin{aligned}
& \left.\begin{array}{l}
C\left(a, b_{t}\right) \geq \lambda-M \cdot v_{1}\left(a, b_{t}\right), \\
C\left(b_{t}, a\right)+\varepsilon \leq \lambda+M \cdot v_{2}\left(a, b_{t}\right), \\
v_{1}\left(a, b_{t}\right)+v_{2}\left(a, b_{t}\right) \leq 1, \\
C\left(b_{h}, a\right) \geq \lambda, \\
C\left(a, b_{h}\right)+\varepsilon \leq \lambda,
\end{array}\right\} \text { if } h<p, \\
& \text { for } t=1, \ldots, h-1 ; \text { if } h>1, \\
& \}
\end{aligned}
$$

where $v_{1}\left(a, b_{t}\right)$ and $v_{2}\left(a, b_{t}\right)$ are binary variables, which guarantee that either $C\left(a, b_{t}\right) \geq \lambda$ or $C\left(b_{t}, a\right)<\lambda$ holds for $t=1, \ldots, h-1$. Note that although original ELECTRE TRI method does not require the DM to specify assignment examples, the above set of constraints may be used in the ordinal regression approach to model such exemplary assignments in case the DM is able to provide some. Then, such a set of constraints needs to be added for all $a^{*} \in A^{R}$ with the proviso that $h$ is replaced by $h^{\mathrm{DM}}\left(a^{*}\right)$. Let us denote the collective set of constraints obtained in this way by $E_{\text {pref }}^{\mathrm{EBP}}$ (for a pessimistic procedure) and $E_{\mathrm{pref}}^{\mathrm{EBO}}$ (for an optimistic procedure).

Specifying the class profiles can be an overwhelming task for a DM. For such decision making situations, there were proposed some outranking methods which require the DM to provide indirect preference information in the form of assignment examples (see, e.g., $[6,16,21])$. An outranking model is said to be consistent with a set of assignment examples if and only if:

$$
\forall a^{*}, b^{*} \in A^{R}, \quad a^{*} S b^{*} \Rightarrow h^{\mathrm{DM}}\left(a^{*}\right) \geq h^{\mathrm{DM}}\left(b^{*}\right),
$$


which can be expressed in a equivalent way as:

$$
\forall a^{*}, b^{*} \in A^{R}, \quad h^{\mathrm{DM}}\left(a^{*}\right)>h^{\mathrm{DM}}\left(b^{*}\right) \Rightarrow b^{*} S^{c} a^{*} .
$$

In terms of an outranking model used in this paper, these requirements can be translated into the following set of constraints:

$$
\left.\begin{array}{l}
C\left(a^{*}, b^{*}\right)+\varepsilon \leq \lambda, \text { for all }\left(a^{*}, b^{*}\right) \in A^{R} \times A^{R}, \\
\text { such that } h^{\mathrm{DM}}\left(a^{*}\right)<h^{\mathrm{DM}}\left(b^{*}\right) .
\end{array}\right\} E_{\text {pref }}^{\mathrm{EC}}
$$

The assignment of the remaining alternatives relies on construction of the model which maintains the desired relations between them and the reference alternatives.

Note that an outranking model based on the concordance index without veto phenomenon is employed in a variety of multiple criteria methods (see, e.g., $[5,16])$. However, within the proposed framework, we could also model the truth and falsity of the outranking relation in a different way. For example, to avoid the need of specification of comparison thresholds by the DM, we could use a simplified model which is in line with the axiomatic approach of Bouyssou and Marchant [3]. Precisely, $\psi_{j}(a, b)=\psi_{j}\left(a_{j}^{*}, a_{j, *}\right)$, if $g_{j}(a) \geq g_{j}(b)$, and $\psi_{j}(a, b)=0$, otherwise. Furthermore, we could take advantage of a more complete model used in ELECTRE ${ }^{\mathrm{GKMS}}$ [7], and require $a S b$ to be true if and only if concordance index $C(a, b)$ is not less than a concordance cutting level $\lambda$, and $a$ is not significantly (by more than a veto threshold) worse than $b$ on any criterion, i.e.:

$$
C(a, b)=\sum_{j=1}^{m} \psi_{j}(a, b) \geq \lambda \text { and } g_{j}(b)-g_{j}(a)<v_{j}, j \in J,
$$

On the contrary, $a$ does not outrank $b$, if either concordance index $C(a, b)$ is less than a concordance cutting level $\lambda$, or there is at least one criterion $g_{j}$ for which $b$ is evaluated better than $a$ by more than a veto threshold $v_{j}$, i.e.:

$$
C(a, b)=\sum_{j=1}^{m} \psi_{j}(a, b)<\lambda+M_{0}(a, b) \text { and } g_{j}(b)-g_{j}(a) \geq v_{j}-M \cdot M_{j}(a, b),
$$

$$
\text { where } M_{j}(a, b) \in\{0,1\}, j=0, \ldots, m \text {, and } \sum_{j=0}^{m} M_{j}(a, b) \leq m \text {. }
$$

Alternatively, we could refer to the credibility degree used in [21], which is defined as follows:

$$
S(a, b)=C(a, b) \cdot\left[1-d^{\max }(a, b)\right],
$$

where $d^{\max }(a, b)=\max _{j \in J} d_{j}(a, b)$, and $d_{j}(a, b)$ is a single-criterion discordance index, which takes a value in the range $[0,1]$, depending on the comparison of the difference $g_{j}(b)-g_{j}(a)$ with the veto threshold $v_{j}$ (for details, see [21]). In the latter variant, the DM would be required to provide precise values for comparison thresholds.

It is also worth stressing that the approach presented in this paper remains valid for the original definition of the credibility degree presented in the ELECTRE TRI method [19,24]. However, the use of such a model in the context of imprecise or indirect preference information is limited by the weak efficiency of non-linear mixed integer programming solvers.

In the following sections, the considered sorting methods based on preference model either in the form of value function or outranking relation, will be called UTADIS-like methods or ELECTRE-like methods, respectively. 
Table 2 Evaluation table for the assessment problem of sales managers
Table 3 The allowed ranges of possible values of indifference and preference thresholds for the assessment problem of sales managers

\begin{tabular}{lrrr}
\hline Alternative & $g_{1}$ & $g_{2}$ & $g_{3}$ \\
\hline Abramov (A) & 100 & 100 & 44 \\
Chen (C) & 85 & 37 & 42 \\
Dall (D) & 72 & 69 & 59 \\
Ellison (E) & 13 & 0 & 68 \\
Furukawa (F) & 0 & 43 & 13 \\
Girouille (G) & 93 & 86 & 31 \\
Hartley (H) & 55 & 23 & 17 \\
Ivashko (I) & 72 & 86 & 25 \\
Johnson (J) & 32 & 70 & 97 \\
Morillo (M) & 29 & 88 & 12 \\
Naray (N) & 41 & 1 & 100 \\
Petersson (P) & 49 & 50 & 0 \\
Stevens (S) & 60 & 9 & 64 \\
Trainini (T) & 15 & 51 &
\end{tabular}

\begin{tabular}{lllll}
\hline & $q_{j, \text { inf }}$ & $q_{j}^{\text {sup }}$ & $p_{j, \text { inf }}$ & $p_{j}^{\text {sup }}$ \\
\hline$g_{1}$ & 2 & 4 & 6 & 9 \\
$g_{2}$ & 1 & 3 & 5 & 7 \\
$g_{3}$ & 1 & 3 & 4 & 5
\end{tabular}

\section{Illustrative example (part 1)}

For the purpose of illustration, let us reconsider the problem discussed in [9], which is about evaluating the performance of company's international sales managers in order to sort them into four classes associated with incentive packages, $\mathrm{C}=\{$ low (LO), lower-middle (LM), upper-middle (UM), high (HI) \}. We will refer to it in the following sections to illustrate different aspects of the proposed framework. We will approach the problem with the UTADIS-like procedure and the ELECTRE-like procedure, both requiring the DM to provide some assignment examples.

Personnel department has considered 15 managers working in different countries. They have taken into consideration three criteria with an increasing direction of preference: sales skills $\left(g_{1}\right)$, territory management skills $\left(g_{2}\right)$, and customer satisfaction $\left(g_{3}\right)$. The evaluations of the alternatives are provided in Table 2 (the abbreviations of the names will be used to refer to the alternatives in the mathematical constraints).

Let us assume that the DM is able to express the following precise judgments about four sales managers: Chen deserves high (HI) incentive package, Ivashko should receive upper-middle (UM) bonus, and the desired classes for Youssef and Trainini are lower-middle (LM) and low (LO), respectively. In order to get a recommendation using the ELECTRE-like method, the DM has to provide preference information consisting of the intervals of possible values of indifference and preference thresholds (see Table 3). 
The set of constraints which defines the set of value functions compatible with the provided assignment examples is the following [to save space, we provide only exemplary monotonicity constraints (two for each marginal value function)]:

$$
\left.\begin{array}{l}
u_{1}\left(g_{1}(\mathrm{Y})\right)-u_{1}\left(g_{1}(\mathrm{~F})\right) \geq 0 ; \ldots ; u_{1}\left(g_{1}(\mathrm{~A})\right)-u_{1}\left(g_{1}(\mathrm{G})\right) \geq 0 \\
u_{2}\left(g_{2}(\mathrm{P})\right)-u_{2}\left(g_{2}(\mathrm{E})\right) \geq 0 ; \ldots ; u_{2}\left(g_{2}(\mathrm{~A})\right)-u_{2}\left(g_{2}(\mathrm{~N})\right) \geq 0 \\
u_{3}\left(g_{3}(\mathrm{~T})\right)-u_{3}\left(g_{3}(\mathrm{P})\right) \geq 0 ; \ldots ; u_{3}\left(g_{3}(\mathrm{~N})\right)-u_{3}\left(g_{3}(\mathrm{~J})\right) \geq 0 \\
u_{1}\left(g_{1}(\mathrm{~F})\right)=0 ; u_{2}\left(g_{2}(\mathrm{E})\right)=0 ; u_{3}\left(g_{3}(\mathrm{P})\right)=0 \\
u_{1}\left(g_{1}(\mathrm{~A})\right)+u_{2}\left(g_{2}(\mathrm{~A})\right)+u_{3}\left(g_{3}(\mathrm{~N})\right)=1 ; \\
U(\mathrm{C}) \geq U(\mathrm{I})+\varepsilon ; U(\mathrm{I}) \geq U(\mathrm{Y})+\varepsilon ; U(\mathrm{Y}) \geq U(\mathrm{~T})+\varepsilon
\end{array}\right\}
$$

The set of constraints which defines the set of compatible outranking models is the following [to save space, we provide only exemplary constraints concerning the shape of marginal concordance functions (one for each constraint type)]:

$$
\begin{aligned}
& \psi_{1}(\mathrm{~A}, \mathrm{~F})+\psi_{2}(\mathrm{~A}, \mathrm{E})+\psi_{3}(\mathrm{~N}, \mathrm{P})=1 ; \\
& \psi_{1}(\mathrm{~A}, \mathrm{~F}) \leq 0.5 ; \psi_{2}(\mathrm{~A}, \mathrm{E}) \leq 0.5 ; \psi_{3}(\mathrm{~N}, \mathrm{P}) \leq 0.5 ; \\
& \psi_{1}(\mathrm{~A}, \mathrm{D}) \geq \psi_{1}(\mathrm{~A}, \mathrm{C}), \text { since } g_{1}(\mathrm{~A})-g_{1}(\mathrm{D})>g_{1}(\mathrm{~A})-g_{1}(\mathrm{C}) \\
& \psi_{1}(\mathrm{~A}, \mathrm{C}) \geq \psi_{1}(\mathrm{~T}, \mathrm{~F}), \quad \text { since } g_{1}(\mathrm{~A})-g_{1}(\mathrm{C})=g_{1}(\mathrm{~T})-g_{1}(\mathrm{~F}) \\
& \psi_{1}(\mathrm{C}, \mathrm{A})=0, \text { since } g_{1}(\mathrm{C})-g_{1}(\mathrm{~A}) \leq-p_{1}^{\text {sup }}=-9 ; \\
& \psi_{1}(\mathrm{~A}, \mathrm{C}) \geq \varepsilon, \quad \text { since } g_{1}(\mathrm{~A})-g_{1}(\mathrm{C})>-p_{1, i n f}=-6 ; \\
& \psi_{1}(\mathrm{~A}, \mathrm{C})=\psi_{1}(\mathrm{~A}, \mathrm{~F}), \quad \text { since } g_{1}(\mathrm{~A})-g_{1}(\mathrm{C}) \geq-q_{1, i n f}=-2 ; \\
& \psi_{1}(\mathrm{C}, \mathrm{A})+\varepsilon \leq \psi_{1}(\mathrm{~A}, \mathrm{~F}), \text { since } g_{1}(\mathrm{C})-g_{1}(\mathrm{~A})<-q_{1}^{\text {sup }}=-4 ; \\
& \psi_{1}(\mathrm{H}, \mathrm{S}) \geq \psi_{1}(\mathrm{~A}, \mathrm{~F}) \cdot 1 / 4, \quad \text { since }-q_{1, \text { inf }}>g_{1}(\mathrm{H})-g_{1}(\mathrm{~S})>-p_{1, i n f} ; \\
& \psi_{1}(\mathrm{H}, \mathrm{S}) \leq \psi_{1}(\mathrm{~A}, \mathrm{~F}) \cdot 4 / 5, \text { since }-q_{1}^{\text {sup }}>g_{1}(\mathrm{H})-g_{1}(\mathrm{~S})>-p_{1}^{\text {sup }} ; \\
& \cdots \\
& C(\mathrm{~T}, \mathrm{Y})+\varepsilon \leq \lambda ; C(\mathrm{~T}, \mathrm{I})+\varepsilon \leq \lambda ; C(\mathrm{~T}, \mathrm{C})+\varepsilon \leq \lambda ; \\
& C(\mathrm{Y}, \mathrm{I})+\varepsilon \leq \lambda ; C(\mathrm{Y}, \mathrm{C})+\varepsilon \leq \lambda ; \\
& C(\mathrm{I}, \mathrm{C})+\varepsilon \leq \lambda
\end{aligned}
$$

Provided preference information is consistent, and, consequently, a set of compatible instances of the preference model is not empty.

\section{Preference information referring to the desired cardinalities of classes}

In this section, we present mathematical models which represent preference information of the DM referring to the desired cardinalities of classes. Note that in all formulations below, $M$ is an auxiliary variable equal to a big positive value, $\varepsilon$ is a small positive value, and $v_{a, C_{h}}$ is a binary variable associated with assignment of alternative $a$ to class $C_{h}$.

Let us first introduce the base model which will be used by different sorting methods. For all these methods, whenever contrary is not explicitly stated, we assume that each alternative $a \in A$ is to be assigned to exactly one class, i.e., for all $a \in A$ :

$$
\sum_{h=1}^{p} v_{a, C_{h}}=1,
$$

and that all exemplary assignments provided by the DM are reproduced, i.e., for all reference alternatives $a^{*} \in A^{R}$ :

$$
v_{a^{*}, C_{h} \mathrm{DM}\left(a^{*}\right)}=1 .
$$


The constraints specific for each method are the following:

- for the UTADIS-like method using class thresholds:

$\left.\begin{array}{l}(U D 1) U(a) \geq b_{h-1}-M \cdot\left(1-v_{a, C_{h}}\right), h=1, \ldots, p, \text { for all } a \in A, \\ (U D 2) U(a)+\varepsilon \leq b_{h}+M \cdot\left(1-v_{a, C_{h}}\right), h=1, \ldots, p, \text { for all } a \in A .\end{array}\right\} E_{\mathrm{card}}^{\mathrm{UD}}$

Constraints $(U D 1)$ and $(U D 2)$ compare $U(a)$ for all alternatives in $A$ with the lower $b_{h-1}$ and upper $b_{h}$ thresholds of class $C_{h}, h=1, \ldots, p$. If a binary variable $v_{a, C_{h}}$, which is associated with the comparison of $a$ with thresholds delimiting the class $C_{h}$, was equal to 0 , then the corresponding constraints would always be satisfied, which is equivalent to elimination of these constraints. On the contrary, if $v_{a, C_{h}}$ was equal to 1 , then alternative $a$ would be assigned to class $C_{h}$, because its comprehensive value $U(a)$ satisfies the following conditions: $b_{h-1} \leq U(a)<b_{h}$. As a result, $U(a)$ is greater than comprehensive values of all reference alternatives assigned by the DM to any class worse than $C_{h}$, and it is smaller than comprehensive values of all reference alternatives assigned by the DM to any class better than $C_{h}$.

- for an example-based value driven sorting procedure:

$$
\left.\begin{array}{c}
(U E 1) U(a)+M \cdot\left(1-v_{a, C_{h}}\right) \geq U(b)+\varepsilon-M \cdot\left(1-v_{b, C_{h-1}}\right), \\
h=2, \ldots, p, \text { for all } a \in A, \text { for all } b \in A \backslash\{a\}, \\
(U E 2) U(a)+\varepsilon-M \cdot\left(1-v_{a, C_{h}}\right) \leq U(b)+M \cdot\left(1-v_{b, C_{h+1}}\right), \\
h=1, \ldots, p-1, \text { for all } a \in A, \text { for all } b \in A \backslash\{a\} .
\end{array}\right\} E_{\mathrm{card}}^{\mathrm{UE}}
$$

Constraints $(U E 1)$ and $(U E 2)$ make sure that, when assigned to class $C_{h}$, the comprehensive value of alternative $a$ is greater than comprehensive values of all alternatives (including non-reference ones) assigned by the procedure to class $C_{h-1}$, and it is less than comprehensive values of all alternatives assigned to class $C_{h+1}$.

If we did not require the final recommendation for each alternative to be a single class assignment, the respective model would be the following:

$\left(U E 1^{\prime}\right) \sum_{h=1}^{p} v_{a}, C_{h} \geq 1$,

$\left(U E 2^{\prime}\right) U(a)+M \cdot\left(1-v_{a, C_{h}}\right) \geq U\left(a_{C_{<h}}\right)+\varepsilon, h=2, \ldots, p$,

for all $a \in A$, for all $a_{C_{<h}} \in A^{R}$, such that $h^{\mathrm{DM}}\left(a_{C_{<h}}\right)<h, \quad \quad E_{\text {card }}^{\mathrm{UE}^{\prime}}$

$\left(U E 3^{\prime}\right) U(a)+\varepsilon-M \cdot\left(1-v_{a, C_{h}}\right) \leq U\left(a_{C_{>h}}\right), h=1, \ldots, p-1$,

for all $a \in A$, for all $a_{C_{>h}} \in A^{R}$, such that $h^{\mathrm{DM}}\left(a_{C_{>h}}\right)>h$.

The explanation of the constraints is analogous to the explanation given in the previous case with the proviso that we collate comprehensive value of $a$ with comprehensive values of reference alternatives only.

- for the ELECTRE-like method with preference information in the form of assignment examples:

(EC1) $C\left(a_{C_{<h}}, a\right)+\varepsilon \leq \lambda+M \cdot\left(1-v_{a, C_{h}}\right), h=2, \ldots, p$, $\left.\begin{array}{l}\text { for all } a \in A \text {, for all } a_{C_{<h}} \in A^{R} \text {, such that } h^{\mathrm{DM}}\left(a_{C_{<h}}\right)<h, \\ (E C 2) C\left(a, a_{C_{>h}}\right)+\varepsilon \leq \lambda+M \cdot\left(1-v_{a, C_{h}}\right), h=1, \ldots, p-1,\end{array}\right\} \quad E_{\text {card }}^{\mathrm{EC}}$ for all $a \in A$, for all $a_{C_{>h}} \in A^{R}$, such that $h^{\mathrm{DM}}\left(a_{C_{>h}}\right)>h$.

Constraints $(E C 1)$ and $(E C 2)$ guarantee that, when assigned to class $C_{h}$, alternative $a$ does not outrank any reference alternative assigned by the DM to a class worse than $C_{h}$, and is not outranked by any reference alternative assigned by the DM to a class better than $C_{h}$, respectively. Consequently, $a$ is either incomparable to each of these alternatives, or it is preferred to (worse than) reference alternatives assigned by the DM to classes worse (better) than $C_{h}$. 
- for a pessimistic rule of the ELECTRE-TRI-like method:

$\left.\begin{array}{l}(E B P 1) C\left(a, b_{h-1}\right) \geq \lambda-M \cdot\left(1-v_{a, C_{h}}\right), h=1, \ldots, p, \text { for all } a \in A, \\ (E B P 2) C\left(a, b_{h}\right)+\varepsilon \leq \lambda+M \cdot\left(1-v_{a, C_{h}}\right) h=1, \ldots, p-1, \text { for all } a \in A .\end{array}\right\} E_{\text {card }}^{\mathrm{EBP}}$

Constraints ( $E B P 1)$ and ( $E B P 2)$ make sure that, when assigned to class $C_{h}$, alternative $a$ outranks all lower profiles of classes not better than $C_{h}$, and does not outrank all lower profiles of classes better than $C_{h}$.

- for an optimistic rule of the ELECTRE-TRI-like method:

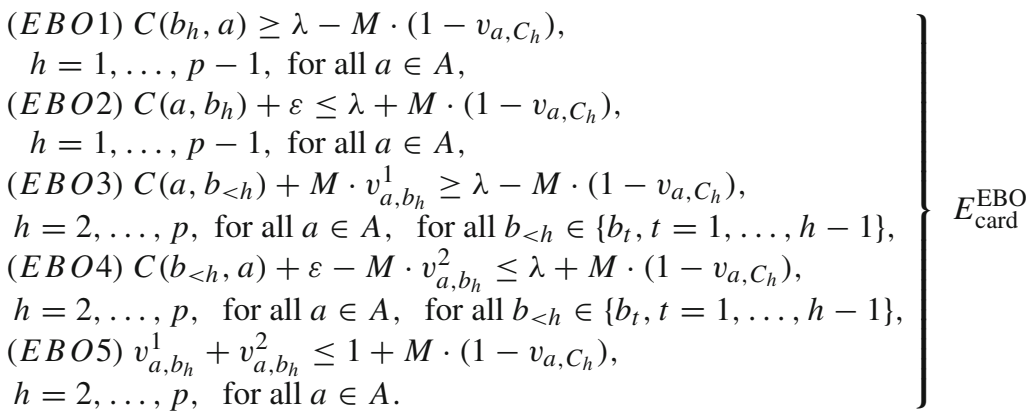

Constraints $(E B O 1)$ and $(E B O 2)$ guarantee that all upper profiles of classes not worse than $C_{h}$ are preferred to alternative $a$ which is assigned to class $C_{h}$. Further, constraints $(E B O 3),(E B O 4)$, and (EBO5) make sure that upper profiles of classes worse than $C_{h}$ are not preferred to $a$ (i.e., for all $b_{t}, t=1, \ldots, h-1$, either $b_{t} S^{c} a$ or $a S b_{t}$, which guarantees that one of the following relations holds: $a \sim b_{t}$ or $a \succ b_{t}$ or $a ? b_{t}$ ).

In Appendix A, we present mathematical models representing assignment rules used in ELECTRE-TRI-C [1], ELECTRE-TRI-nC [2] and FlowSort [20] outranking methods. These models can further incorporate the preference information referring to the desired cardinalities of classes. This proves generality of the proposed methodology.

Let us pass now to the modeling of the desired cardinalities of classes in terms of constraints which will be added to the previously defined mathematical models. These constraints are the same irrespective of the sorting method under consideration:

- $C_{h}$ should contain at least $C_{h}^{\min }$ and at most $C_{h}^{\max }$ alternatives, with $C_{h}^{\min } \leq C_{h}^{\max }$ :

$$
\left.\begin{array}{l}
\text { (CL) } \sum_{a \in A} v_{a, C_{h}} \geq C_{h}^{\min } \\
\text { (CU) } \sum_{a \in A} v_{a, C_{h}} \leq C_{h}^{\max }
\end{array}\right\}
$$

Note that if $v_{a, C_{h}}$ was equal to 0 , then we relax all conditions which are necessary to assign $a$ to class $C_{h}$. On the contrary, if $v_{a, C_{h}}$ was equal to 1 , then $a$ is assigned to class $C_{h}$, since all constraints which are necessary to place $a$ in $C_{h}$ are satisfied. Constraint $(C L)$ ensures that there are at least $C_{h}^{\min }$ alternatives assigned to $C_{h}$, whereas constraint $(C U)$ guarantees that there are at most $C_{h}^{\max }$ alternatives assigned to $C_{h}$. Obviously, we can use either $(C L)$ or $(C U)$ only to model the requirements concerning the lower or upper bound on the cardinality of class $C_{h}$, respectively.

- $C_{h}$ should contain at least $C_{h}^{\text {min-perc }}$ (in \%) and at most $C_{h}^{\max -p e r c}$ (in \%) of alternatives, with $C_{h}^{\text {min-perc }} \leq C_{h}^{\text {max-perc,": }}$

$$
\left.\begin{array}{l}
(C L \%) \sum_{a \in A} v_{a, C_{h}} \geq\left\lceil C_{h}^{\text {min-perc }} \cdot n\right\rceil \\
(C U \%) \sum_{a \in A} v_{a, C_{h}} \leq\left\lfloor C_{h}^{\max -\text { perc }} \cdot n\right\rfloor .
\end{array}\right\}
$$


If the DM expressed the desired cardinality of class $C_{h}$ in terms of the percentage of the whole set of alternatives $A$ (e.g., 5, $10 \%$, a quarter, one third, or half of all alternatives), such statement would be modeled analogously to situation when the cardinality is provided explicitly. However, if $C_{h}^{\text {min-perc }}\left(C_{h}^{\text {max-perc }}\right.$ ) of $n$ was not equal to a natural number, we need to guarantee that the smallest (the greatest) number of alternatives which may be assigned to $C_{h}$ is equal to $\left\lceil C_{h}^{\text {min-perc }} \cdot n\right\rceil\left(\left\lfloor C_{h}^{\text {max-perc }} \cdot n\right\rfloor\right)$.

- the range of $k$ contiguous classes $C_{t}, t=h, h+1, \ldots, h+k-1$, should contain at least $C_{h, k}^{\min }$ and at most $C_{h, k}^{\max }$ alternatives, with $C_{h, k}^{\min } \leq C_{h, k}^{\max }$ and $k \geq 1$ :

$$
\left.\begin{array}{l}
(U C L) \sum_{t=h}^{h+k-1} \sum_{a \in A} v_{a, C_{t}} \geq C_{h, k}^{\min }, \\
(U C U) \sum_{t=h}^{h+k-1} \sum_{a \in A} v_{a, C_{t}} \leq C_{h, k}^{\max } .
\end{array}\right\}
$$

This type of statement could be used to express the requirements with respect to the cardinality of class union $C_{h}^{\geq}$(classes not worse than $C_{h}$, i.e., a set of the $(p-h+1)$ best classes) and $C_{h}^{\leq}$(classes not better than $C_{h}$, i.e., a set of the $h$ worst classes). Obviously, the statements expressing the cardinality of the union of classes with respect to the percentage of the whole set of alternatives could be modeled analogously to the previous case.

- relative comparisons of cardinalities of different classes, e.g., class $C_{h}$ should contain not less alternatives than class $C_{l}$, i.e.:

$$
\sum_{a \in A} v_{a, C_{h}} \geq \sum_{a \in A} v_{a, C_{l}}
$$

This kind of inequality could also be used to model the requirement that the number of alternatives assigned to each class should be more or less the same, i.e. they should not differ by more than a given number.

Let us denote the set of constraints modeling the statements of the DM referring to the cardinalities of classes by $E_{\text {card }}^{\text {req }}$.

\section{Illustrative example (part 2)}

For our illustrative example, let us additionally assume that the DM needs to obey some funding limits and, thus, (s)he defines some requirements with respect to the numbers of sales managers who should be assigned to the best and to the worst class. Precisely, the sorting method should assign from 2 to 4 alternatives to class HI, and from 3 to 5 alternatives to class LO. Translating these requirements into mathematical constraints requires the use of binary variable $v_{a, C_{h}}$ for each $a \in A$ and $h \in H$. For example, a binary variable $v_{\mathrm{A}, C_{H I}}$ is associated with the assignment of Abramov (A) to class HI.

The constraints which guarantee that all assignment examples and all desired cardinalities of classes are reproduced is the following:

$$
\left.\begin{array}{l}
v_{\mathrm{C}, C_{H I}}=1 ; v_{\mathrm{I}, C_{U M}}=1 ; v_{\mathrm{Y}, C_{L M}}=1 ; v_{\mathrm{T}, C_{L O}}=1 ; \\
v_{\mathrm{A}, C_{L O}}+v_{\mathrm{A}, C_{L M}}+v_{\mathrm{A}, C_{U M}}+v_{\mathrm{A}, C_{H I}}=1 ; \ldots ; \\
v_{\mathrm{Y}, C_{L O}}+v_{\mathrm{Y}, C_{L M}}+v_{\mathrm{Y}, C_{U M}}+v_{\mathrm{Y}, C_{H I}}=1 ; \\
v_{\mathrm{A}, C_{L O}}+v_{\mathrm{C}, C_{L O}}+\cdots+v_{\mathrm{T}, C_{L O}}+v_{\mathrm{Y}, C_{L O}} \geq 3 ; \\
v_{\mathrm{A}, C_{L O}}+v_{\mathrm{C}, C_{L O}}+\cdots+v_{\mathrm{T}, C_{L O}}+v_{\mathrm{Y}, C_{L O}} \leq 5 ; \\
v_{\mathrm{A}, C_{H I}}+v_{\mathrm{C}, C_{H I}}+\cdots+v_{\mathrm{T}, C_{H I}}+v_{\mathrm{Y}, C_{H I}} \geq 2 \\
v_{\mathrm{A}, C_{H I}}+v_{\mathrm{C}, C_{H I}}+\cdots+v_{\mathrm{T}, C_{H I}}+v_{\mathrm{Y}, C_{H I}} \leq 4 .
\end{array}\right\}
$$


Further, the constraints specific for the UTADIS-like method are the following (to save space, for both UTADIS- and ELECTRE-like methods we provide explicitly only exemplary constraints regarding assignment of Abramov (A) to different classes):

$$
\left.\begin{array}{l}
U(\mathrm{~A})+M \cdot\left(1-v_{\mathrm{A}, C_{L M}}\right) \geq U(\mathrm{C})+\varepsilon-M \cdot\left(1-v_{\mathrm{C}, C_{L O}}\right) ; \ldots ; \\
U(\mathrm{~A})+M \cdot\left(1-v_{\mathrm{A}, C_{L M}}\right) \geq U(\mathrm{Y})+\varepsilon-M \cdot\left(1-v_{\mathrm{Y}, C_{L O}}\right) ; \\
U(\mathrm{~A})+M \cdot\left(1-v_{\mathrm{A}, C_{U M}}\right) \geq U(\mathrm{C})+\varepsilon-M \cdot\left(1-v_{\mathrm{C}, C_{L M}}\right) ; \ldots ; \\
U(\mathrm{~A})+M \cdot\left(1-v_{\mathrm{A}, C_{U M}}\right) \geq U(\mathrm{Y})+\varepsilon-M \cdot\left(1-v_{\mathrm{Y}, C_{L M}}\right) ; \\
U(\mathrm{~A})+M \cdot\left(1-v_{\mathrm{A}, C_{H I}}\right) \geq U(\mathrm{C})+\varepsilon-M \cdot\left(1-v_{\mathrm{C}, C_{U M}}\right) ; \ldots ; \\
U(\mathrm{~A})+M \cdot\left(1-v_{\mathrm{A}, C_{H I}}\right) \geq U(\mathrm{Y})+\varepsilon-M \cdot\left(1-v_{\mathrm{Y}, C_{U M}}\right) ; \\
U(\mathrm{~A})+\varepsilon-M \cdot\left(1-v_{\mathrm{A}, C_{L O}}\right) \leq U(\mathrm{C})+M \cdot\left(1-v_{\mathrm{C}, C_{L M}}\right) ; \ldots ; \\
U(\mathrm{~A})+\varepsilon-M \cdot\left(1-v_{\mathrm{A}, C_{L O}}\right) \leq U(\mathrm{Y})+M \cdot\left(1-v_{\mathrm{Y}, C_{L M}}\right) ; \\
U(\mathrm{~A})+\varepsilon-M \cdot\left(1-v_{\mathrm{A}, C_{L M}}\right) \leq U(\mathrm{C})+M \cdot\left(1-v_{\mathrm{C}, C_{U M}}\right) ; \ldots ; \\
U(\mathrm{~A})+\varepsilon-M \cdot\left(1-v_{\mathrm{A}, C_{L M}}\right) \leq U(\mathrm{Y})+M \cdot\left(1-v_{\mathrm{Y}, C_{U M}}\right) ; \\
U(\mathrm{~A})+\varepsilon-M \cdot\left(1-v_{\mathrm{A}, C_{U M}}\right) \leq U(\mathrm{C})+M \cdot\left(1-v_{\mathrm{C}, C_{H I}}\right) ; \ldots ; \\
U(\mathrm{~A})+\varepsilon-M \cdot\left(1-v_{\mathrm{A}, C_{U M}}\right) \leq U(\mathrm{Y})+M \cdot\left(1-v_{\mathrm{Y}, C_{H I}}\right) .
\end{array}\right\}
$$

The constraints specific for the ELECTRE-like method are the following:

$$
\left.\begin{array}{l}
C(\mathrm{~A}, \mathrm{Y})+\varepsilon \leq \lambda+M \cdot\left(1-v_{\mathrm{A}, C_{L O}}\right) \\
C(\mathrm{~A}, \mathrm{I})+\varepsilon \leq \lambda+M \cdot\left(1-v_{\mathrm{A}, C_{L O}}\right) ; \\
C(\mathrm{~A}, \mathrm{C})+\varepsilon \leq \lambda+M \cdot\left(1-v_{\mathrm{A}, C_{L O}}\right) \\
C(\mathrm{~T}, \mathrm{~A})+\varepsilon \leq \lambda+M \cdot\left(1-v_{\mathrm{A}, C_{L M}}\right) \\
C(\mathrm{~A}, \mathrm{Y})+\varepsilon \leq \lambda+M \cdot\left(1-v_{\mathrm{A}, C_{L M}}\right) \\
C(\mathrm{~A}, \mathrm{I})+\varepsilon \leq \lambda+M \cdot\left(1-v_{\mathrm{A}, C_{L M}}\right) \\
C(\mathrm{~T}, \mathrm{~A})+\varepsilon \leq \lambda+M \cdot\left(1-v_{\mathrm{A}, C_{U M}}\right) ; \\
C(\mathrm{Y}, \mathrm{A})+\varepsilon \leq \lambda+M \cdot\left(1-v_{\mathrm{A}, C_{U M}}\right) \\
C(\mathrm{~A}, \mathrm{I})+\varepsilon \leq \lambda+M \cdot\left(1-v_{\mathrm{A}, C_{U M}}\right) ; \\
C(\mathrm{~T}, \mathrm{~A})+\varepsilon \leq \lambda+M \cdot\left(1-v_{A, C_{H I}}\right) ; \\
C(\mathrm{Y}, \mathrm{A})+\varepsilon \leq \lambda+M \cdot\left(1-v_{A, C_{H I}}\right) ; \\
C(\mathrm{C}, \mathrm{A})+\varepsilon \leq \lambda+M \cdot\left(1-v_{A, C_{H I}}\right)
\end{array}\right\}
$$

\section{Multiple criteria sorting methods respecting requirements referring to the desired cardinalities of classes}

Let us define the set of instances of the preference model compatible with the preference information provided by the DM for each sorting method under consideration. For the value-driven sorting procedures, a compatible value function satisfies the following set of constraints:

- for the UTADIS-like procedure $E^{\mathrm{UD}}$ consisting of $E_{\mathrm{base}}^{\mathrm{UD}}, E_{\mathrm{pref}}^{\mathrm{UD}}, E_{\mathrm{card}}^{\mathrm{UD}}$, and $E_{\text {card }}^{\mathrm{req}}$;

- for an example-based procedure $E^{\mathrm{UE}}$ consisting of $E_{\mathrm{base}}^{\mathrm{UE}}, E_{\mathrm{pref}}^{\mathrm{UE}}, E_{\mathrm{card}}^{\mathrm{UE}}$, and $E_{\text {card }}^{\mathrm{req}}$.

For the ELECTRE-like procedures, a compatible outranking model is defined by concordance indices $C(a, b)$, concordance cutting level $\lambda$, indifference $q_{j}$, and preference $p_{j}$ thresholds, and weights $k_{j}$, for all $a, b \in A, j \in J$, satisfying the following set of constraints:

- for the ELECTRE-like method with preference information in the form of assignment examples $-E^{\mathrm{EC}}$ consisting of: $E_{\mathrm{base}}^{\mathrm{EL}}, E_{\mathrm{pref}}^{\mathrm{EC}}, E_{\text {card }}^{\mathrm{EC}}$, and $E_{\text {card }}^{\mathrm{req}}$;

- for a pessimistic rule of the ELECTRE-TRI-like method- $E^{E B P}$ consisting of: $E_{\text {base }}^{\mathrm{EL}}, E_{\mathrm{pref}}^{\mathrm{EBP}}, E_{\text {card }}^{\mathrm{EBP}}$, and $E_{\text {card }}^{\mathrm{req}}$; 
- for an optimistic rule of the ELECTRE-TRI-like method- $E^{\mathrm{EBO}}$ consisting of: $E_{\mathrm{base}}^{\mathrm{EL}}$, $E_{\text {pref }}^{\mathrm{EBO}}, E_{\mathrm{card}}^{\mathrm{EBO}}$, and $E_{\text {card }}^{\mathrm{req}}$.

In order to verify that the set of compatible instances of the preference model (set of value functions $\mathcal{U}_{A^{R}}$ or set of outranking relations $\mathcal{S}_{A^{R}}$ ) is not empty, we consider the following mixed integer linear program (MILP):

\section{Maximize: $\varepsilon$, subject to $E^{A^{R}}$,}

where $E^{A^{R}}$ is equal to $E^{\mathrm{UD}}$ or $E^{\mathrm{UE}}$ or $E^{\mathrm{EC}}$ or $E^{\mathrm{EBP}}$ or $E^{\mathrm{EBO}}$, depending on the sorting method under consideration. Let us denote by $\varepsilon^{*}$ the maximal value of $\varepsilon$ obtained from the solution of the above MILP problem, i.e., $\varepsilon^{*}=\max (\varepsilon)$, subject to $E^{A^{R}}$. We conclude that the set of compatible instances of the preference model is not empty, if $E^{A^{R}}$ is feasible and $\varepsilon^{*}>0$.

If the set of compatible instances of the preference model is not empty, there is usually more than one compatible instance. For the UTADIS-like method, a reasonable way of selecting a single value function consists in maximization of the distance between the values of alternatives placed in each class and the respective class thresholds; in this way, one gets as "sharp" discrimination between classes as possible. This requires consideration of the set of constraints $E^{\mathrm{UD}^{\prime}}$, which is obtained by replacing $E_{\mathrm{card}}^{\mathrm{UD}}$ in $E^{\mathrm{UD}}$ by the following set of constraints, which define the difference between comprehensive values of alternatives and class thresholds, using additional slack variables $\varepsilon(C), \varepsilon_{*}\left(C_{h}\right), \varepsilon^{*}\left(C_{h}\right), \varepsilon_{*}\left(a, C_{h}\right)$, and $\varepsilon^{*}\left(a, C_{h}\right), h=1, \ldots, p$, and $a \in A$ :

$$
\left.\begin{array}{l}
U(a)+\varepsilon_{*}\left(a, C_{h}\right) \geq b_{h-1}-M \cdot\left(1-v_{a, C_{h}}\right), h=1, \ldots, p, \text { for all } a \in A, \\
\varepsilon_{*}\left(a, C_{h}\right) \geq \varepsilon_{*}\left(C_{h}\right), h=1, \ldots, p, \text { for all } a \in A, \\
\varepsilon_{*}\left(C_{h}\right) \varepsilon(C), h=1, \ldots, p, \\
U(a)+\varepsilon^{*}\left(a, b_{h}\right) \leq b_{h}+M \cdot\left(1-v_{a, C_{h}}\right), h=1, \ldots, p, \text { for all } a \in A, \quad \\
\varepsilon^{*}\left(a, C_{h}\right) \geq \varepsilon^{*}\left(C_{h}\right), h=1, \ldots, p, \text { for all } a \in A, \\
\varepsilon^{*}\left(C_{h}\right) \geq \varepsilon(C), h=1, \ldots, p, \\
\varepsilon(C) \geq 0 .
\end{array}\right\} \quad E_{\mathrm{post}-\mathrm{Upt}}^{\mathrm{UD}}
$$

In order to achieve sharp discrimination, we can conduct the following optimizations in a lexicographic order:

- maximization of the minimal distance of the comprehensive values of alternatives from the respective class thresholds, i.e.: Maximize : $\varepsilon(C)$, subject to $E^{\mathrm{UD}^{\prime}}$;

- maximization of the sum of minimal distances of the comprehensive values of alternatives from each class threshold, i.e.: Maximize : $\sum_{h=1}^{p}\left(\varepsilon_{*}\left(C_{h}\right)+\varepsilon^{*}\left(C_{h}\right)\right.$ ) (with the proviso that $\varepsilon(C)$ is equal to the optimal value obtained in the previous point);

- maximization of the sum of elementary distances of the comprehensive values of alternatives from the corresponding class thresholds, i.e.: Maximize : $\sum_{a \in A} \sum_{h=1}^{p}\left(\varepsilon_{*}\left(a, C_{h}\right)+\right.$ $\varepsilon^{*}\left(a, C_{h}\right)$ ) (with the proviso that $\varepsilon_{*}\left(C_{h}\right)$ and $\varepsilon^{*}\left(C_{h}\right)$ are equal to the optimal value obtained in the previous point).

Note that for an example-based value-driven sorting procedure, we should maximize the distance between comprehensive values of the alternatives placed in different classes, rather than between the values of alternatives placed in each class and the respective class thresholds.

For the ELECTRE-like methods, we could proceed analogously and emphasize the consequences of assigning the alternatives to particular classes, by making the result of the corresponding concordance tests as sharp as possible. Let us present it on the example of the ELECTRE-like method with preference information in the form of assignment examples. 
The selection of a single instance of the compatible outranking relation for the ELECTRETRI-like methods can be conducted analogously. We will consider the set of constraints $E^{\mathrm{EC}^{\prime}}$, which is obtained by replacing $E_{\text {card }}^{\mathrm{EC}}$ in $E^{\mathrm{EC}}$ by the following set of constraints with additional slack variables $\varepsilon(C), \varepsilon_{*}\left(C_{<h}\right), \varepsilon^{*}\left(C_{>h}\right), \varepsilon_{*}\left(a, C_{<h}\right)$, and $\varepsilon^{*}\left(a, C_{>h}\right), h=1, \ldots, p$, and $a \in A$ :

$$
\begin{aligned}
& C\left(a_{C_{<h}}, a\right)+\varepsilon_{*}\left(a, C_{<h}\right) \leq \lambda+M \cdot\left(1-v_{a, C_{h}}\right), h=2, \ldots, p \\
& \text { for all } a \in A \text {, for all } a_{C_{<h}} \in A^{R} \text {, such that } h^{\mathrm{DM}}\left(a_{C_{<h}}\right)<h \text {, } \\
& \varepsilon_{*}\left(a, C_{<h}\right) \geq \varepsilon_{*}\left(C_{<h}\right), h=2, \ldots, p \text {, for all } a \in A \\
& \varepsilon_{*}\left(C_{<h}\right) \geq \varepsilon(C), h=2, \ldots, p, \\
& C\left(a, a_{C_{>h}}\right)+\varepsilon^{*}\left(a, C_{>h}\right) \leq \lambda+M \cdot\left(1-v_{a, C_{h}}\right) h=1, \ldots, p-1, \quad E_{\text {post-opt }}^{\mathrm{EC}} \\
& \text { for all } a \in A \text {, for all } a_{C_{. h}} \in A^{R} \text {, such that } h^{\mathrm{DM}}\left(a_{C_{>h}}\right)>h \text {, } \\
& \varepsilon_{*}\left(a, C_{>h}\right) \geq \varepsilon_{*}\left(C_{>h}\right), h=1, \ldots, p-1, \text { for all } a \in A \text {, } \\
& \varepsilon_{*}\left(C_{>h}\right) \geq \varepsilon(C), h=1, \ldots, p-1 \text {, } \\
& \varepsilon(C) \geq 0 \text {. }
\end{aligned}
$$

Then, analogously to the UTADIS-like method, we could first maximize $\varepsilon(C)$, then the sum of $\varepsilon_{*}\left(C_{<h}\right)$ and $\varepsilon_{*}\left(C_{>h}\right)$ for $h=1, \ldots, p$, and finally the sum of elementary differences $\varepsilon_{*}\left(a, C_{<h}\right)$ and $\varepsilon_{*}\left(a, C_{>h}\right)$ for $a \in A$ and $h=1, \ldots, p$.

Alternatively, as suggested by Köksalan et al. [15], we could try to force alternatives in higher-level classes to outrank alternatives in lower-level classes, and minimize the number of pairs of alternatives for which this requirement is not satisfied. For this reason, we introduce new binary variables $w_{a, a^{*}}$ and $w_{a^{*}, a}$ for all $a \in A \backslash A^{R}$ and all $a^{*} \in A^{R}$. If $w_{a, a^{*}}$ was equal to 1 , then the desired outranking relation between $a$ and $a^{*}$ would be violated. Consequently, to select an outranking model with the mini number of such violations, we solve the following MILP problem:

$$
\text { Minimize : } \sum_{a \in A \backslash A^{R}} \sum_{a^{*} \in A^{R}} w_{a, a^{*}}+w_{a^{*}, a}
$$

subject to:

$$
\begin{aligned}
& E^{E C} \\
& C\left(a, a_{C_{<h}}\right) \geq \lambda-M \cdot\left(1-v_{a, C_{h}}\right)-M \cdot w_{a, a_{C_{<h}}}, h=2, \ldots, p, \\
& \text { for all } a \in A \backslash A^{R}, \text { for all } a_{C_{<h}} \in A^{R}, \text { such that } h^{\mathrm{DM}}\left(a_{C_{<h}}\right)<h \\
& C\left(a_{C_{>h}}, a\right) \geq \lambda-M \cdot\left(1-v_{a, C_{h}}\right)-M \cdot w_{a_{C_{>}, a}}, h=1, \ldots, p-1, \\
& \text { for all } a \in A \backslash A^{R}, \text { for all } a_{C_{>h}} \in A^{R} \text {, such that } h^{\mathrm{DM}}\left(a_{C_{>h}}\right)>h .
\end{aligned}
$$

Note that instead of choosing any particular instance of the preference model, we could also take into account all compatible instances and determine the set of possible assignments for each alternative $a \in A$. To check whether $a$ could be possibly assigned to a class $C_{h}$, it is sufficient to assume in the set of constraints $E^{A^{R}}$ that $v_{a, C_{h}}$ is equal to 1 , and, subsequently, check whether the resulting set of constraints is feasible. This needs to be done for all $a \in A$ for each particular class $C_{h}, h=1, \ldots, p$. This approach may be particularly useful in the process of incremental specification of assignment examples by the DM. Knowing the set of possible assignments for each alternative, the DM may wish to change the assignment suggested by the method at a current stage of interaction-such a change will be possible when the set of compatible instances of the preference model in the next iteration will not be empty. 
Table 4 The final assignments for the assessment problem of sales managers

\begin{tabular}{ll}
\hline Class & Number of alternatives: assigned alternatives \\
\hline UTADIS-like method & \\
HI & 3: Abramov, Chen, Girouille \\
UM & 5: Dall, Hartley, Ivashko, Johnson, Naray \\
LM & 4: Ellison, Petersson, Stevens, Youssef \\
LO & 3: Furukawa, Morillo, Trainini \\
ELECTRE-like method & \\
HI & 4: Abramov, Chen, Dall, Girouille \\
UM & 5: Ivashko, Johnson, Morillo, Naray, Stevens \\
LM & 3: Furukawa, Hartley, Youssef \\
LO & 3: Ellison, Petersson, Trainini \\
\hline
\end{tabular}

As suggested in [18], in order to support specification of the desired cardinalities of classes $C_{h}, h=1, \ldots, p$, one may compute the extreme possible cardinalities given a set of compatible preference model instances. In the framework of DIS-CARD, this can be achieved by solving the following problem:

$$
\text { Maximize (Minimize): } \sum_{a \in A} v_{a, C_{h}} \text {, subject to } E^{A^{R}} \text {. }
$$

Note that thus obtained maximal and minimal cardinalities of each class are certainly, respectively, not greater and not less than the corresponding requirements specified by the DM. Obviously, the number of alternatives that are possibly assigned to $C_{h}$ when considering the set of compatible model instances, may be, in general, greater than the maximal cardinality of $C_{h}$.

\section{Illustrative example (part 3)}

In order to choose a single instance which would underly the final recommendation for our illustrative example, for the UTADIS-like method, we maximize the slack variables to obtain as sharp discrimination as possible, whereas for the ELECTRE-like method, we try to force alternatives in higher-level classes to outrank alternatives in lower-level classes and we minimize the number of violations of this requirement. The corresponding assignments obtained with the use of both sorting methods are presented in Table 4.

The assignments of reference alternatives are reproduced and the number of alternatives assigned to the best and to the worst class are within the pre-defined limits. Obviously, the recommendation worked out for some non-reference alternatives is different in case of both methods. In particular, there are seven such alternatives for which the assignment is different, whereas for the remaining four alternatives the class is the same for UTADIS-like and ELECTRE-like methods. The differences stem from:

- Fundamental differences in assumptions of these methods. Let us remind that the purpose of UTADIS-like methods is to model the decision making situation with an overall value function, and to assign a score to each alternative. Such a score reflects a comprehensive value of the alternative on all criteria considered jointly, and drives the assignment of alternatives to decision classes. The additive value function used in UTADIS-like methods is a compensatory aggregation model admitting that a loss on any criterion can be compensated by a gain on another criterion. When it comes to ELECTRE-like methods, their preference structure is based on a binary outranking relation between particular alternatives and reference profiles characterizing decision classes. The outranking relation is a 
non-compensatory aggregation model. Moreover, it does not assign any numerical score to each alternative. Its exploitation has purely ordinal character.

- Different optimization criteria taken into account when selecting a single compatible instance of the preference model.

- Resulting different priorities assigned to the evaluation criteria in the selected instances of the preference model underlying the final recommendation. Precisely, for the UTADIS-like method the maximal difference between comprehensive values of alternatives and class thresholds is equal to 0.142 . The greatest share in the comprehensive values is assigned to criterion $g_{1}$, whereas the least share is assigned to criterion $g_{2}$. Consequently, sales managers with the best evaluations on $g_{1}$ (e.g., Abramov, Girouille) are assigned to class HI, whereas alternatives with relatively poor evaluations on $g_{1}$ are attributed worse incentive packages. When it comes to the ELECTRE-like method, the number of violations of the desired outranking relation between alternatives in higher-level classes and alternatives in lower-level classes is equal to 12 (pairs (Youssed, Ellison), (Ivashko, Furukawa), (Chen, Johnson), etc.). The greatest weights are assigned to $g_{1}$ and $g_{2}$, which favors alternatives with at least moderately good evaluations on these criteria.

Obviously, the DM may wish to continue the solution process. Precisely, (s)he may add some assignment examples, contradicting the recommendation obtained at the current stage for some non-reference alternatives. Moreover, (s)he may add some requirements with respect to the desired cardinalities of classes UM or LM, or make the requirements which have been already provided for classes $\mathrm{HI}$ and LO more precise.

\section{Conclusions}

In this paper, we introduced a new approach to multiple criteria sorting problems which allows incorporation of preference information concerning the desired cardinalities of classes. The main motivation for this proposal comes from the common use of such requirements in practical situations, the willingness of the DMs to refer to the bounds on the cardinalities of classes, and some undesired properties of the recommendation worked out with the use of traditional methods, which do not admit any control of the cardinalities of classes. We proposed some mixed-integer programming models, which allow incorporation of the preference information of this type. Moreover, we have shown that the introduced approach is valid for any type of employed preference model. Precisely, we have proposed some value driven sorting procedures, and some ELECTRE-like methods which are modeling the DM's preferences with an outranking relation and require the DM to define the classes with the use of either class profiles, or characteristic alternatives, or assignment examples.

Further research directions include thorough experimental analysis of the introduced approach, and its application to some real world problems e.g., in the field of finance. Moreover, this type of preference information may be used in disaggregation procedures which aim to infer precise parameters for the ELECTRE-like methods from imprecise or indirect preferences of the DM.

Acknowledgments The authors would like to thank the anonymous reviewers for their helpful comments and suggestions. The first author wishes to acknowledge financial support from the Poznan University of Technology, grant no. 91-516/DS-MLODA KADRA.

Open Access This article is distributed under the terms of the Creative Commons Attribution License which permits any use, distribution, and reproduction in any medium, provided the original author(s) and the source are credited. 


\section{Appendix A: Handling of preference information referring to the desired cardinalities of classes in ELECTRE-TRI-C, ELECTRE-TRI-nC and FlowSort methods}

In this section, we recall the ELECTRE-TRI-C, ELECTRE-TRI-nC and FlowSort methods, and we formulate mathematical models representing their assignment rules. These models can further incorporate the preference information referring to the desired cardinalities of classes.

\section{A.1 ELECTRE-TRI-C}

ELECTRE-TRI-C is an outranking method for multiple criteria sorting problems in which each class is defined by a single characteristic reference alternative instead of a pair of class profiles. Let $B=\left\{b_{0}, b_{1}, \ldots, b_{p}, b_{p+1}\right\}$ denote the set of $(p+2)$ characteristic alternatives, where $b_{0}$ and $b_{p+1}$ have the worst and the best evaluations on all criteria, respectively, whereas $b_{t}, t=1, \ldots, p$, are alternatives provided by the DM, which are characteristic for particular classes and fulfill several intuitive conditions defined in [1]. In particular, $b_{t}$ need to dominate $b_{t-1}, t=1, \ldots, p+1$. ELECTRE-TRI-C uses two assignment rules, called descending and ascending rules. They employ a selecting function $\rho\left(a, b_{t}\right)$. The authors suggest that $\rho\left(a, b_{t}\right)=\min \left\{C\left(a, b_{t}\right), C\left(b_{t}, a\right)\right\}$ fulfills the desired properties of such a function. The descending rule, compares $a$ successively to $b_{h}$, for $h=p, \ldots, 0$, for a particular concordance cutting level $\lambda$, seeking for the first characteristic alternative $b_{h}$, such that $C\left(a, b_{h}\right) \geq \lambda$. If $h=p$, then it assigns $a$ to $C_{p}$; if $h=0$, then $a$ is placed in $C_{1}$; otherwise $a$ is assigned to $C_{h}$ if $\rho\left(a, b_{h}\right)>\rho\left(a, b_{h+1}\right)$, or to $C_{h+1}$ if $\rho\left(a, b_{h+1}\right) \geq \rho\left(a, b_{h}\right)$. On the contrary, an ascending rule compares $a$ successively to $b_{t}, h=1, \ldots, p+1$, seeking for the first characteristic alternative $b_{h}$, such that $C\left(b_{h}, a\right) \geq \lambda$. If $h=1$, then $a$ is assigned to $C_{1}$; if $h=p+1$, then $a$ is placed in $C_{p}$; otherwise $a$ is assigned to $C_{h}$ if $\rho\left(a, b_{h}\right)>\rho\left(a, b_{h-1}\right)$, or to $C_{h-1}$ if $\rho\left(a, b_{h-1}\right) \geq \rho\left(a, b_{h}\right)$.

The models which allow subsequent incorporation of the preference information referring to the desired cardinalities of classes are the following:

- for the descending rule of the ELECTRE-TRI-C-like method:

$$
\left.\begin{array}{l}
(E C D 1) C\left(a, b_{h}\right)+M \cdot v_{a, b_{h}}^{1} \geq \lambda-M \cdot\left(1-v_{a, C_{h}}\right), \\
\quad h=1, \ldots, p, \text { for all } a \in A, \\
(E C D 2) C\left(a, b_{h+1}\right)+\varepsilon-M \cdot v_{a, b_{h}}^{1} \leq \lambda+M \cdot\left(1-v_{a, C_{h}}\right), \\
\quad h=1, \ldots, p-1, \text { for all } a \in A, \\
(E C D 3) C\left(b_{h}, a\right)+M \cdot v_{a, b_{h}}^{1} \geq C\left(a, b_{h+1}\right)+\varepsilon-M \cdot\left(1-v_{a, C_{h}}\right), \\
\quad h=1, \ldots, p-1, \text { for all } a \in A, \\
(E C D 4) C\left(a, b_{h-1}\right)+M \cdot v_{a, b_{h}}^{2} \geq \lambda-M \cdot\left(1-v_{a, C_{h}}\right), \\
\quad h=1, \ldots, p, \text { for all } a \in A, \\
(E C D 5) C\left(a, b_{h}\right)+\varepsilon-M \cdot v_{a, b_{h}}^{2} \leq \lambda+M \cdot\left(1-v_{a, C_{h}}\right), \\
\quad h=2, \ldots, p, \text { for all } a \in A, \\
(E C D 6) C\left(a, b_{h}\right)+M \cdot v_{a, b_{h}}^{2} \geq C\left(b_{h-1}, a\right)-M \cdot\left(1-v_{a, C_{h}}\right), \\
\quad h=2, \ldots, p, \text { for all } a \in A, \\
(E C D 7) v_{a, b_{h}}^{1}+v_{a, b_{h}}^{2} \geq 1-M \cdot\left(1-v_{a, C_{h}}\right), h=1, \ldots, p, \text { for all } a \in A, \\
(E C D 8) v_{a, b_{h}}^{1}+v_{a, b_{h}}^{2} \leq 1+M \cdot\left(1-v_{a, C_{h}}\right), h=1, \ldots, p, \text { for all } a \in A .
\end{array}\right\} E_{\mathrm{card}}^{\mathrm{ECD}}
$$

Constraints (ECD1) and (ECD2) make sure that, when assigned to class $C_{h}$, alternative $a$ outranks all alternatives which are characteristic for classes not better than $C_{h}$, 
and does not outrank all alternatives characteristic for classes better than $C_{h}$. Constraint (ECD3) guarantees satisfying the condition necessary for $a$ to be assigned to $C_{h}$ rather than to $C_{h+1}$. On the other hand, $(E C D 4)$ and (ECD5) make sure that $a$ outranks all characteristic alternatives for classes worse than $C_{h}$, and does not outrank all characteristic alternatives for classes not worse than $C_{h}$. Constraint (ECD6) guarantees that when ( $E C D 4)$ and (ECD5) are satisfied, $a$ would be assigned to $C_{h}$ rather than to $C_{h-1}$. Constraints $(E C D 7)$ and (ECD8) employ binary variables $v_{a, b_{h}}^{1}$ and $v_{a, b_{h}}^{2}$, guaranteeing that either $(E C D 1),(E C D 2)$, and $(E C D 3)$, or (ECD4), (ECD5), and (ECD6) will hold. As a result, one of the two possible sets of conditions for the assignment of $a$ to $C_{h}$ will be satisfied.

- for the ascending rule of the ELECTRE-TRI-C-like method:

$$
\left.\begin{array}{l}
(E C A 1) C\left(b_{h}, a\right)+M \cdot v_{a, b_{h}}^{1} \geq \lambda-M \cdot\left(1-v_{a, C_{h}}\right), \\
\quad h=1, \ldots, p, \text { for all } a \in A . \\
(E C A 2) C\left(b_{h-1}, a\right)+\varepsilon-M \cdot v_{a, b_{h}}^{1} \leq \lambda+M \cdot\left(1-v_{a, C_{h}}\right), \\
\quad h=2, \ldots, p, \text { for all } a \in A, \\
(E C A 3) C\left(a, b_{h}\right)+M \cdot v_{a, b_{h}}^{1} \geq C\left(b_{h-1}, a\right)+\varepsilon-M \cdot\left(1-v_{a, C_{h}}\right), \\
\quad h=2, \ldots, p, \text { for all } a \in A, \\
(E C A 4) C\left(b_{h+1}, a\right)+M \cdot v_{a, b_{h}}^{2} \geq \lambda-M \cdot\left(1-v_{a, C_{h}}\right), \\
\quad h=1, \ldots, p, \text { for all } a \in A, \\
(E C A 5) C\left(b_{h}, a\right)+\varepsilon-M \cdot v_{a, b_{h}}^{2} \leq \lambda+M \cdot\left(1-v_{a, C_{h}}\right), \\
\quad h=1, \ldots, p-1, \text { for all } a \in A . \\
(E C A 6) C\left(b_{h}, a\right)+M \cdot v_{a, b_{h}}^{2} \geq C\left(a, b_{h+1}\right)-M \cdot\left(1-v_{a, C_{h}}\right), \\
\quad h=1, \ldots, p-1, \text { for all } a \in A, \\
(E C A 7) v_{a, b_{h}}^{1}+v_{a, b_{h}}^{2} \geq 1-M \cdot\left(1-v_{a, C_{h}}\right), h=1, \ldots, p, \text { for all } a \in A, \\
(E C A 8) v_{a, b_{h}}^{1}+v_{a, b_{h}}^{2} \leq 1+M \cdot\left(1-v_{a, C_{h}}\right), h=1, \ldots, p, \text { for all } a \in A .
\end{array}\right\} E_{\text {card }}^{\mathrm{ECA}}
$$

The explanation of the constraints is analogous to the explanation given for the descending rule, i.e. assignment of alternative $a$ to class $C_{h}$ is conditioned by satisfying either (ECA1), (ECA2), and (ECA3), or (ECA4), (ECA5), and (ECA6).

\section{A.2 ELECTRE-TRI-nC}

The ELECTRE-TRI-nC method [2] generalizes ELECTRE-TRI-C by admitting classes $C_{h}, h=1, \ldots, p$, to be characterized by several typical reference alternatives $B_{h}=\left\{b_{h}^{r}, r=\right.$ $\left.1, \ldots, m_{h}\right\}$ instead of a single central profile only. The method considers categorical credibility (concordance) indices defined as follows:

$$
\begin{gathered}
C\left(a, B_{h}\right)=\max _{r=1, \ldots, m_{h}}\left\{C\left(a, b_{h}^{r}\right)\right\}, \\
C\left(B_{h}, a\right)=\max _{r=1, \ldots, m_{h}}\left\{C\left(b_{h}^{r}, a\right)\right\} .
\end{gathered}
$$

Note that these categorical indices could be defined in the following way with the use of binary variables $v\left(a, b_{h}^{r}\right)$, for $a \in A, h=1, \ldots, p$, and $r=1, \ldots, m_{h}$ :

$$
\begin{aligned}
& C\left(a, B_{h}\right) \geq C\left(a, b_{h}^{r}\right), h=1, \ldots, p, \text { for } r=1, \ldots, m_{h}, \\
& C\left(a, B_{h}\right) \leq C\left(a, b_{h}^{r}\right)+v\left(a, b_{h}^{r}\right), h=1, \ldots, p, \text { for } r=1, \ldots, m_{h}, \\
& \sum_{r=1}^{m_{h}} v\left(a, b_{h}^{r}\right) \leq m_{h}-1, h=1, \ldots, p, \\
& C\left(B_{h}, a\right) \geq C\left(b_{h}^{r}, a\right), h=1, \ldots, p, \text { for } r=1, \ldots, m_{h}, \\
& C\left(B_{h}, a\right) \leq C\left(b_{h}^{r}, a\right)+v\left(b_{h}^{r}, a\right), h=1, \ldots, p, \text { for } r=1, \ldots, m_{h}, \\
& \sum_{r=1}^{m_{h}} v\left(b_{h}^{r}, a\right) \leq m_{h}-1, h=1, \ldots, p .
\end{aligned}
$$


In this way, $C\left(a, B_{h}\right)\left(C\left(B_{h}, a\right)\right)$ is required to be not less than concordance indices $C\left(a, b_{h}^{r}\right)\left(C\left(b_{h}^{r}, a\right)\right), r=1, \ldots, m_{h}$, and to be equal to at least one of them, which, in fact, needs to be maximal.

The descending and ascending assignment rules are analogical as in the ELECTRE-TRI$\mathrm{C}$ method, but refer to the categorical indices rather than traditional credibility indices. To save space, we present a model which allows subsequent incorporation of the preference information referring to the desired cardinalities of classes for the descending rule only:

$(E n C D 1) C\left(a, B_{h}\right)+M \cdot v_{a, B_{h}}^{1} \geq \lambda-M \cdot\left(1-v_{a, C_{h}}\right)$,

$h=1, \ldots, p$, for all $a \in A$,

$(E n C D 2) C\left(a, B_{h+1}\right)+\varepsilon-M \cdot v_{a, B_{h}}^{1} \leq \lambda+M \cdot\left(1-v_{a, C_{h}}\right)$,

$h=1, \ldots, p-1$, for all $a \in A$,

$(E n C D 3) C\left(B_{h}, a\right)+M \cdot v_{a, B_{h}}^{1} \geq C\left(a, B_{h+1}\right)+\varepsilon-M \cdot\left(1-v_{a, C_{h}}\right)$,

$h=1, \ldots, p-1$, for all $a \in A$,

$(E n C D 4) C\left(a, B_{h+1}\right)+\varepsilon-M \cdot v_{a, B_{h}}^{1} \leq C\left(a, B_{h}\right)+M \cdot\left(1-v_{a, C_{h}}\right)$,

$h=1, \ldots, p-1$, for all $a \in A$,

$(E n C D 5) C\left(a, B_{h-1}\right)+M \cdot v_{a, B_{h}}^{2} \geq \lambda-M \cdot\left(1-v_{a, C_{h}}\right)$,

$h=1, \ldots, p$, for all $a \in A$,

$(E n C D 6) C\left(a, B_{h}\right)+\varepsilon-M \cdot v_{a, B_{h}}^{2} \leq \lambda+M \cdot\left(1-v_{a, C_{h}}\right)$,

$h=2, \ldots, p$, for all $a \in A$,

$(E n C D 7) C\left(a, B_{h}\right)+M \cdot v_{a, B_{h}}^{2}+M \cdot v_{a, B_{h}}^{x} \geq C\left(B_{h-1}, a\right)-M \cdot\left(1-v_{a, C_{h}}\right)$, $h=2, \ldots, p$, for all $a \in A$,

$(E n C D 8) C\left(a, B_{h}\right)+M \cdot v_{a, B_{h}}^{2}+M \cdot v_{a, B_{h}}^{y} \geq C\left(a, B_{h-1}\right)-M \cdot\left(1-v_{a, C_{h}}\right)$, $h=2, \ldots, p$, for all $a \in A$,

$(E n C D 9) C\left(a, B_{h}\right)-M \cdot v_{a, B_{h}}^{2}-M \cdot v_{a, B_{h}}^{y} \leq C\left(a, B_{h-1}\right)+M \cdot\left(1-v_{a, C_{h}}\right)$, $h=2, \ldots, p$, for all $a \in A$,

$(E n C D 10) v_{a, B_{h}}^{x}+v_{a, B_{h}}^{y} \leq 1+M \cdot\left(1-v_{a, C_{h}}\right), h=1, \ldots, p$, for all $a \in A$,

$(E n C D 11) v_{a, B_{h}}^{1}+v_{a, B_{h}}^{2} \geq 1-M \cdot\left(1-v_{a, C_{h}}\right), h=1, \ldots, p$, for all $a \in A$,

$(E n C D 12) v_{a, B_{h}}^{1}+v_{a, B_{h}}^{2} \leq 1+M \cdot\left(1-v_{a, C_{h}}\right), h=1, \ldots, p$, for all $a \in A$.

The explanation of the role of the constraints is analogical as for the ELECTRE-TRI-C method. Constraint (EnCD3) and (EnCD4) guarantee satisfying the conditions necessary for $a$ to be assigned to $C_{h}$ rather than to $C_{h+1}$, whereas constraints (EnCD7)-(EnCD10) guarantee that $a$ would be assigned to $C_{h}$ rather than to $C_{h-1}$. For detailed explanation of these conditions, see [2].

\section{A.3 FlowSort}

FlowSort is a PROMETHEE-like method for multiple criteria sorting problems [20]. The assignment of a given alternative is based on its comparison with either limiting profiles or central profiles in terms of the positive (incoming), negative (leaving), and/or net outranking flows. Let us focus on the procedure which employs limiting profiles $B=\left\{b_{0}, b_{1}, \ldots, b_{p}\right\}$, such that $b_{t}$ dominates $b_{t-1}, t=1, \ldots, p$, and net outranking flows defined as follows for any $a \in A \cup B$ (let us denote by $R(a)=B \cup\{a\}$, where $a$ is an alternative to be assigned): 


$$
\begin{aligned}
\Phi_{R(a)}(a) & =\Phi_{R(a)}^{+}(a)-\Phi_{R(a)}^{-}(a)=1 /(|R(a)|-1) \sum_{b \in R(a)} \pi(a, b)-\pi(b, a) \\
& =1 /(|R(a)|-1) \sum_{b \in R(a)} \sum_{j=1}^{m} \pi_{j}(a, b)-\pi_{j}(b, a),
\end{aligned}
$$

where $\pi_{j}, j \in j$, is a preference function, indicating the degree to which $a$ is preferred to $b$ on criterion $g_{j}$ with respect to the provided indifference $q_{j}$ and preference $p_{j}$ thresholds. In [12], we have generalized the family of PROMETHEE methods so that it accounts for imprecise preference information in the form of admissible ranges of indifference and preference thresholds (see notation in Sect. 2.2). Moreover, we defined preference functions as general non-decreasing ones, and not only of arbitrarily chosen shape. Precisely, they need to satisfy the following set of conditions:

$$
\left.\begin{array}{l}
\sum_{j=1}^{m} \pi_{j}\left(a_{j}^{*}, a_{j, *}\right)=1:\left(g_{j}\left(a_{j}^{*}\right)=x_{j}^{n_{j}(A)}\right) \text { and }\left(g_{j}\left(a_{j, *}\right)=x_{j}^{1}\right) \\
\quad \text { with } a_{j}^{*}, a_{j, *} \in A, j \in J, \\
\pi_{j}\left(a_{j}^{*}, a_{j, *}\right) \leq 0.5, j \in J, \\
\text { for all }(a, b, c, d) \in A \times A \times A \times A, j \in J: \\
\quad \pi_{j}(a, b) \geq \pi_{j}(c, d) \text { if } g_{j}(a)-g_{j}(b)>g_{j}(c)-g_{j}(d), \\
\pi_{j}(a, b)=\pi_{j}(c, d) \text { if } g_{j}(a)-g_{j}(b)=g_{j}(c)-g_{j}(d), \\
\text { for all }(a, b) \in A \times A, j \in J: \\
\quad \pi_{j}(a, b)=0 \text { if } g_{j}(a)-g_{j}(b) \leq q_{j, i n f}, \\
\pi_{j}(a, b) \geq \varepsilon \text { if } g_{j}(a)-g_{j}(b)>q_{j}^{\text {sup }}, \\
\pi_{j}(a, b)+\varepsilon \leq \pi_{j}\left(a_{j}^{*}, a_{j, *}\right) \text { if } g_{j}(a)-g_{j}(b)<p_{j, i n f}, \\
\pi_{j}(a, b)=\pi_{j}\left(a_{j}^{*}, a_{j, *}\right) \text { if } g_{j}(a)-g_{j}(b) \geq p_{j}^{\text {sup }} .
\end{array}\right\} E_{\mathrm{base}}^{\mathrm{PR}}
$$

These conditions ensure normalization, monotonicity, and desired shape of the preference functions in the areas of indifference, weak preference, and strict preference (for detailed explanation, see [12]) Obviously, in case of a FlowSort-like method in all above formulations one should consider set $A \cup B$ rather than $A$ only. The model which allows subsequent incorporation of the preference information referring to the desired cardinalities of classes is the following:

$\left.\begin{array}{l}(P R 1) \Phi_{R(a)}(a) \geq \Phi_{R(a)}\left(b_{h-1}\right)-M \cdot\left(1-v_{a, C_{h}}\right), h=1, \ldots, p, \text { for all } a \in A, \\ (P R 2) \Phi_{R(a)}(a)+\varepsilon \leq \Phi_{R(a)}\left(b_{h}\right)+M \cdot\left(1-v_{a, C_{h}}\right), h=1, \ldots, p, \text { for all } a \in A .\end{array}\right\} E_{\mathrm{card}}^{\mathrm{PR}}$

The models for the case of using central profiles (rather than limit profiles) and/or considering positive and negative flows separately (rather than net outranking flows) could be formulated analogously.

\section{References}

1. Almeida-Dias, J., Figueira, J.R., Roy, B.: Electre Tri-C: a multiple criteria sorting method based on characteristic reference actions. Eur. J. Oper. Res. 204(3), 565-580 (2010)

2. Almeida-Dias, J., Figueira, J.R., Roy, B.: A multiple criteria sorting method where each category is characterized by several reference actions: the electre Tri-nC method. Eur. J. Oper. Res. 217(3), 567579 (2012)

3. Bouyssou, D., Marchant, T.: An axiomatic approach to noncompensatory sorting methods in MCDM, I: the case of two categories. Eur. J. Oper. Res. 178(1), 217-245 (2007) 
4. Devaud, J.M., Groussaud, G., Jacquet-Lagreze, E.: UTADIS: Une methode de construction de fonctions d'utilite additives rendant compte de jugements globaux. In: Proceedings of the European Working Group on MCDA, Bochum, Germany (1980)

5. Dias, L., Mousseau, V., Figueira, J., Clímaco, J.: An aggregation/disaggregation approach to obtain robust conclusions with ELECTRE TRI. Eur. J. Oper. Res. 138(2), 332-348 (2002)

6. Fernandez, E., Navarro, J.: A new approach to multi-criteria sorting based on fuzzy outranking relations: the THESEUS method. Eur. J. Oper. Res. 213(2), 405-413 (2011)

7. Greco, S., Kadziński, M., Mousseau, V., Słowiński, R.: ELECTREGKMS : Robust ordinal regression for outranking methods. Eur. J. Oper. Res. 214(1), 118-135 (2011)

8. Greco, S., Kadziński, M., Mousseau, V., Słowiński, R.: Robust ordinal regression for multiple criteria group decision problems: UTA ${ }^{\text {GMS }}$ _GROUP and UTADIS ${ }^{\text {GMS }}$ _GROUP. Decis. Support Syst. 52(3), 549-561 (2012)

9. Greco, S., Kadziński, M., Słowiński, R.: Selection of a representative value function in robust multiple criteria sorting. Comput. Oper. Res. 38(11), 1620-1637 (2011)

10. Greco, S., Mousseau, V., Słowiński, R.: Multiple criteria sorting with a set of additive value functions. Eur. J. Oper. Res. 207(4), 1455-1470 (2010)

11. Kadziński, M., Greco, S., Słowiński, R.: Selection of a representative value function for robust ordinal regression in group decision making. Group Decis. Negot. 1-34 (2011). doi:10.1007/s10726-011-9277-z

12. Kadziński, M., Greco, S., Słowiński, R.: Extreme ranking analysis in robust ordinal regression. Omega 40(4), 488-501 (2012)

13. Kadziński, M., Greco, S., Słowiński, R.: Selection of a representative set of parameters for robust ordinal regression outranking methods. Comput. Oper. Res. 39(11), 2500-2519 (2010)

14. Keeney, R.L., Raiffa, H.: Decisions with Multiple Objectives: Preferences and Value Trade-Offs. Cambridge University Press, Cambridge (1976)

15. Köksalan, M., Bilgin Özpeynirci, S.: An interactive sorting method for additive utility functions. Comput. Oper. Res. 36(9), 2565-2572 (2009)

16. Köksalan, M., Mousseau, V., Özpeynirci, O., Bilgin Özpeynirci, S.: An outranking-based approach for assigning alternatives to ordered classes. Nav. Res. Logist. 56(1), 74-85 (2009)

17. Köksalan, M., Ulu, C.: An interactive approach for placing alternatives in preference classes. Eur. J. Oper. Res. 144, 429-439 (2003)

18. Mousseau, V., Dias, L.C., Figueira, J.: On the notion of category size in multiple criteria sorting models. Cahier du LAMSADE 205, Université Paris-Dauphine, Paris, France (2003)

19. Mousseau, V., Słowiński, R.: Inferring an ELECTRE TRI model from assignment examples. J. Glob. Optim. 12(2), 157-174 (1998)

20. Nemery, P., Lamboray, C.: Flow Sort: a flow-based sorting method with limiting or central profiles. TOP 16, 90-113 (2008)

21. Rocha, C., Dias, L.C.: An algorithm for ordinal sorting based on ELECTRE with categories defined by examples. J. Glob. Optim. 42, 255-277 (2008)

22. Roy, B.: The outranking approach and the foundations of ELECTRE methods. In: Bana e Costa, C.A. (ed.) Readings in Multiple Criteria Decision Aid, pp. 155-183. Springer, Berlin (1990)

23. Srinivasan, V.: Linear programming computational procedures for ordinal regression. J. ACM 23(3), 475487 (1976)

24. Yu, W.: ELECTRE TRI: aspects méthodologiques et manuel d'utilisation. Document du LAMSADE no 74, Université Paris-Dauphine (1992)

25. Zopounidis, C., Doumpos, M.: PREFDIS: a multicriteria decision support system for sorting decision problems. Comput. Oper. Res. 27(7-8), 779-797 (2000)

26. Zopounidis, C., Doumpos, M.: Multicriteria classification and sorting methods: A literature review. Eur. J. Oper. Res. 138, 229-246 (2002) 